Document downloaded from:

http://hdl.handle.net/10251/37518

This paper must be cited as:

Catalá Bolós, A.; García Sanjuan, F.; Jaén Martínez, FJ.; Mocholi Agües, JA. (2012). TangiWheel: A widget for manipulating collections on tabletop displays supporting hybrid Input modality. Journal of Computer Science and Technology. 27(4):811-829. doi:10.1007/s11390-012-1266-4.

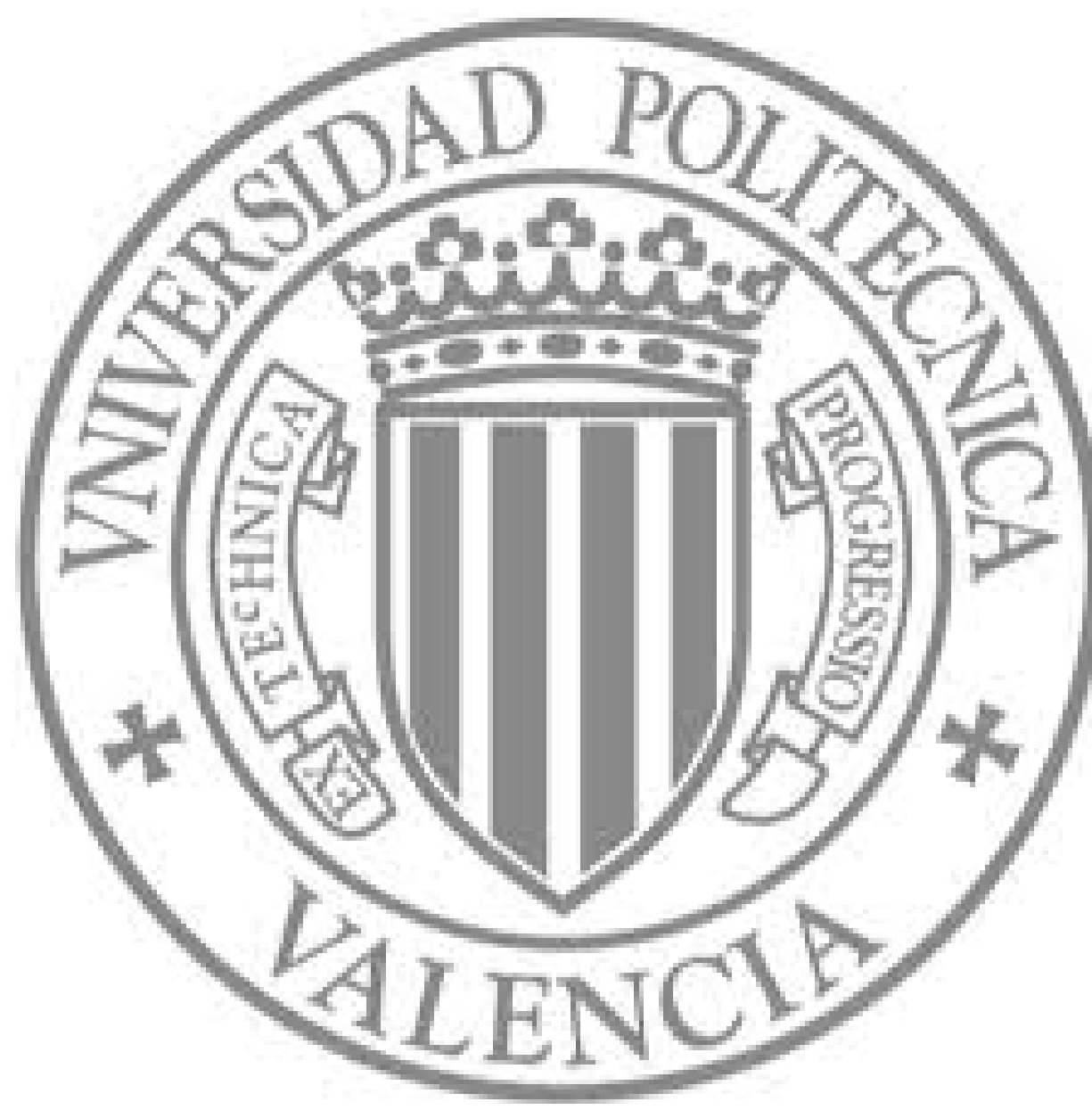

The final publication is available at

http://link.springer.com/article/10.1007/s11390-012-1266-4

Copyright Springer Verlag (Germany) 


\title{
TangiWheel: A Widget for Manipulating Collections on Tabletop Displays Supporting Hybrid Input Modality*
}

\author{
Alejandro Catala, Member, ACM, IEEE, Fernando Garcia-Sanjuan, Javier Jaen, Jose A. Mocholi \\ ISSI, Departamento de Sistemas Informáticos y Computación, Universitat Politènica de València, Camí de \\ Vera s/n, 46022, Valencia, Spain \\ E-mail: \{acatala, fegarcia, fjaen, jmocholi\}@dsic.upv.es
}

\begin{abstract}
In this paper we present TangiWheel, a collection manipulation widget for tabletop displays. Our implementation is flexible, allowing either multi-touch or tangible interaction, or even a hybrid scheme to better suit user choice and convenience. Different TangiWheel aspects and features are compared with other existing widgets for collection manipulation. The study reveals that TangiWheel is the first proposal to support a hybrid input modality with high resemblance levels between touch and tangible interaction styles. Several experiments were conducted to evaluate the techniques used in each input scheme for a better understanding of tangible surface interfaces in complex tasks performed by a single user (e.g. involving a typical master-slave exploration pattern). The results showed that tangibles perform significantly better than fingers, despite dealing with a greater number of interactions, in situations that require a high number of acquisitions and basic manipulation tasks such as establishing location and orientation. However, when users have to perform multiple exploration and selection operations that do not require previous basic manipulation tasks, for instance when collections are fixed in the interface layout, touch input is significantly better in terms of required time and number of actions. Finally, when a more elastic collection layout or more complex additional insertion or displacement operations are needed, the hybrid and tangible approaches clearly outperform finger-based interactions.
\end{abstract}

Keywords Tangible, Interactive surface, Multi-touch, Tangible User Interface (TUI), Tabletop displays, Experimentation

\footnotetext{
${ }^{*}$ PAPER CLASSIFICATION

Supported by the Ministry of Education of Spain under project Grant No. TIN2010-20488. A. Catalá is supported by a FPU fellowship with reference AP2006-00181.
} 
Catala A, Garcia-Sanjuan F, Jaen J et al. TangiWheel: A Widget for Manipulating Collections on Tabletop Displays Supporting Hybrid Input Modality. JOURNAL OF COMPUTER SCIENCE AND TECHNOLOGY Vol.(No.):1-end Mon. Year

\section{Introduction}

Multi-touch and tangible surface interfaces have become popular in recent years. While multi-touch tabletops allow collective and multiple interaction with virtual objects through finger contact on the surface, tangible tabletops expand multi-touch input capabilities by using specially designed tangible objects. These interaction mechanisms provide more intuitive performance and interaction. Several innovative applications such as Reactable [1], IntuPaint [2] or Multi-Touch VirtualGlobe [3] have been developed in the research community to demonstrate the new capabilities of tabletop interfaces.

The growing success of these new interaction mechanisms has also fostered a number of comparative studies. In one of the seminal studies, Fitzmaurice \& Buxton conducted an empirical comparison of spatial-multiplexed versus time-multiplexed input schemes [4]. The spatial-multiplexed conditions used specialized input devices, such as a rotor, a brick, a stretchable square and ruler, as well as several generic puck and brick sets, whereas the time-multiplexed condition only used one puck and brick. The study concludes that spatial-multiplexed conditions are superior to time-multiplexed input schemes in terms of tracking error.

In the same line of research, another experimental comparison was carried out by Tuddenham, who explored the benefits of Tangible User Interfaces (TUIs) versus multi-touch input, measuring the performance in terms of time and tracking error [5]. Since the explored TUI elements can be used in tangible tabletops, many of their benefits could be applied to tangible surfaces, given that these are a specific TUI type. The comparison explores simple but common basic interface actions found in many multi-touch and tangible surface interfaces focused on acquisition and manipulation issues. This is an important contribution because it provides designers with the information needed to decide between TUIs and multi-touch input on interactive surfaces. It also assesses the value of the tangible element to the user in this type of system, especially in the case of the TUI elements explored in the study, whose physical shape and size perfectly match the characteristics of the virtual counterparts.

Another empirical study of interest is reported in [6]. It also compares touch-versus-tangible input in a tabletop, but focuses on general manipulations on box-like elements, such as walls and shelves. The study reports on an experiment which asked subjects to reproduce models of walls and shelves as fast and accurately as possible. In this task, requiring spatial layout on a tabletop, tangible input took much less time but it was slightly less accurate than touch.

However, despite these research and development efforts, further studies are needed to obtain a better understanding of interactions with more complex types of controls to enable the develop- 
ment of applications that address the more challenging user tabletop requirements. Among the many interactions that will have to be addressed on tabletops, there is a subset of special interest to us which is widely present in everyday desktop-applications: the manipulation of collections. Collections are typically supported on desktop applications by list or menu widgets. Lists generally contain a potentially unlimited collection of items, which are typically application-domain entities (i.e. data), whereas menus normally contain application commands or option selections. Menus are usually fixed, or have a limited collection, generally always-visible and linked to a point on the user interface. On tabletops, however, lists and menus do not have a predetermined form and quite often the difference between them is somewhat blurred. Firstly, neither of them is fixed to a point on the tabletop because menu options are usually attached to information elements scattered across the surface. Secondly, both must support a 360-degree interaction style, so that the interface is not oriented towards one user when several are collaborating on the same surface, or at least a way to manage this issue. Thirdly, both must provide effective mechanisms for the search and selection of elements so that the tabletop surface is not overloaded when multiple search and selection processes (on different lists/menus) are taking place simultaneously. Thus, a unified approach for both types of collections seems attainable on tabletops, and a study of the more complex interactions related to collections manipulation for both existing interaction styles (i.e. multi-touch and tangible) is a necessary step forward with respect to previous comparative studies that only take into consideration simple widgets and actions.

In this paper, we present, on the one hand, a unified interaction widget for generic collection navigation and item selection on tabletops following a comparative analysis of other research work, and on the other, an empirical comparison of pure multi-touch input versus tangible-input along with a hybrid input condition. The final goal is thus to propose a unified widget to manipulate collections homogeneously in tangible only, multi-touch only and also in a combination of both interaction styles while presenting the design decisions that were taken and studying the effectiveness of the supported interaction styles.

The rest of the paper is organized as follows. Section 2 describes related studies and compares their different aspects and features. Section 3 then introduces the proposed widget by means of a discussion of the aforementioned aspects. In Section 4 the experimental context for the study and its main objectives are described. Sections 5, 6, 7 and 8 report on the experiments conducted in the course of this work, with our conclusions and intentions for further research.

\section{Related Work}

The work described in this paper was influenced by a range of different studies. Firstly, the seminal work of Fitzmaurice, Ishii and Buxton on graspables (tangibles) [7], as well as Ishii \& Ullmer's vision of “Tangible bits” [8], have dramatically impacted the concept of the tangible surface interface, in which direct tangible manip- 
ulation is performed through physical handlers or bricks. Other studies have described the use of phicons for containing, transporting and manipulating digital objects [9].

Shen et. al presented $\mathrm{CoR}^{2} \mathrm{D}$, a virtual control for interactive pop-ups, which allows the visualization and launch of commands [10]. $\operatorname{CoR}^{2} \mathrm{D}$ menu items can be moved and rotated by means of virtual handlers strategically attached to the menu items. This flexibility greatly helps to achieve desirable features such as occlusion, reach, establishing context on cluttered display, readability, and concurrent, coordinated multiuser interaction. However the unsystematic layout of menu items on the surface makes this approach unsuitable for the simultaneous manipulation of multiple collections by different users, since the visual feedback from the different items can cause confusion.

As pointed out in [11], even though a great deal of work has been carried out on menus in recent years, menu systems for multi-touch platforms have received little attention. Efforts have been focused on improving performance of large mouse-based menus [12], [13] or pen-based interfaces, where marking menus have clearly taken the lead in their different approaches and variants [14], [15]. These generally provide support for hierarchical collection exploration but not for virtually unlimited items, despite the effort to substantially extend the size of the collection.

Patten et. al describe a series of interaction techniques for the exploration and selection of items [16]. These techniques primarily consist of an approach based on two-handed interaction via pucks, one of which represents the item to be modified and the other is the modifier. An important feature is the visualization of items as pie menus. Another technique is characterized by the use of non-circular "floating menus". The activation of both menu techniques is bound to specific sensitive areas or hotspots limiting flexible access to menu instantiation, although tangibles are always required.

Weiss et. al [17] present a set of active silicone peripherals, such as knobs, keyboards and buttons, which can easily be stuck at any point on the surface. This is a more elaborate approach than the phicons, and also provides more physical feedback, which improves the performance of interaction tasks. This study explores the use of a knob to manipulate pie menus and concludes that for simple tasks this more physical approach provides significantly quicker task completion than a purely virtual approach. However, it neither considers nor explores the use of cascading menus, which would perhaps be useful to support more complex navigation and other tasks, as a consequence of relying solely on physical peripherals.

Lepinsky et. al evaluate a marking menu specifically designed for multi-touch platforms [11] based on directional chording gestures. His experiments showed that multi-touch marking menus perform faster than traditional hierarchical marking menus. 
Hancock et. al present a radial control that supports 2D information exploration [18]. It is implemented in two versions, a direct-touch two-handed interaction implementation and a tangible knob with an embedded trackball. Both approaches require quite different manipulation techniques. While the tangible device only requires one hand, the touch-based control needs two hands. This device is specifically designed to explore collections and no other function is considered, such as indistinctly containing collections or single items. The part of the study dealing with data exploration focuses on the use of both versions in terms of perception of ease-of-use. It concludes that the tangible approach is easier to use for navigation and sharing information.

Hilliges et. al present a tangible rotatory tabletop device for browsing digital photo collections [19]. It uses a novel helix representation for the photos, which are anchored around the device itself. A pen is used to support application-specific manipulations, such as image subsets. Photohelix is oriented towards time-based visualization, which allows for a sequential spiral-based visualization of elements, but there is no support for nested sub-collections consistently.
Stacked half-pie menus are presented by Hesselmann et. al for navigation in nested hierarchic data structures optimized for touch-based interaction on interactive tabletops [20]. This design is especially interesting because it is conceptually unlimited in terms of menu depth and breadth while maintaining menu form. It is controlled entirely by means of touch and turn gestures, with no need to use a pen, gloves or any other tool. It also tackles the multi-touch occlusion problem by using only half-pies and sticking them to one side of the tabletop. This approach, however, only allows for parallel touches if several leaf items are at the same level, otherwise, interaction will be sequential. The control is conceived as a non-collaborative (single-user) menu and it is fixed to a specific location.

In the Tangible Jukebox [21] music navigation and management is carried out by means of a pie-based widget controlled by tangibles and fingers. It uses physical cards to virtually store a music collection. When a card is placed on the table, a set of items is deployed around it, composing a virtual wheel that the user can spin to visualize all the menu-level content. Several set operations can be performed by combining specific physical cards accordingly. 
These related works can be described in terms of features for a more systematic comparison. These features are broadly related to main aspects concerning the generality of the approach, data organization features, input methods and multiuser support. Table 1 shows a description of related works.

The generic-purpose aspect indicates whether the proposal is to be used in either a generic or specific purpose domain. The data organization aspect is concerned with features on how data are supported and organized. Length and nested hierarchy support are related to the capability for breadth and depth exploration, respectively. The length supported is reported at three levels: short (S), when a short collection of elements are intended; large (L), when a larger, though still lim- ited, set of elements can be contained; and finally virtually unlimited (U), when the length could be considered large or even infinite. The nested hierarchy support simply indicates whether or not the proposal supports hierarchical collections consistently across several depth levels. The feature massive collection display indicates whether several collections can be displayed and manipulated at the same time. Dynamic hierarchies refers to the ability to establish hierarchies of collections dynamically, in contrast to only statically predefined collection hierarchies. Spatial compactness indicates whether the proposal includes a characteristic to deal with spatial limitations in its design. Finally, visual layout reports on the visual arrangement of the items in the collection.

Regarding the relevant features describing the

Table 1. Feature based comparison between related works.

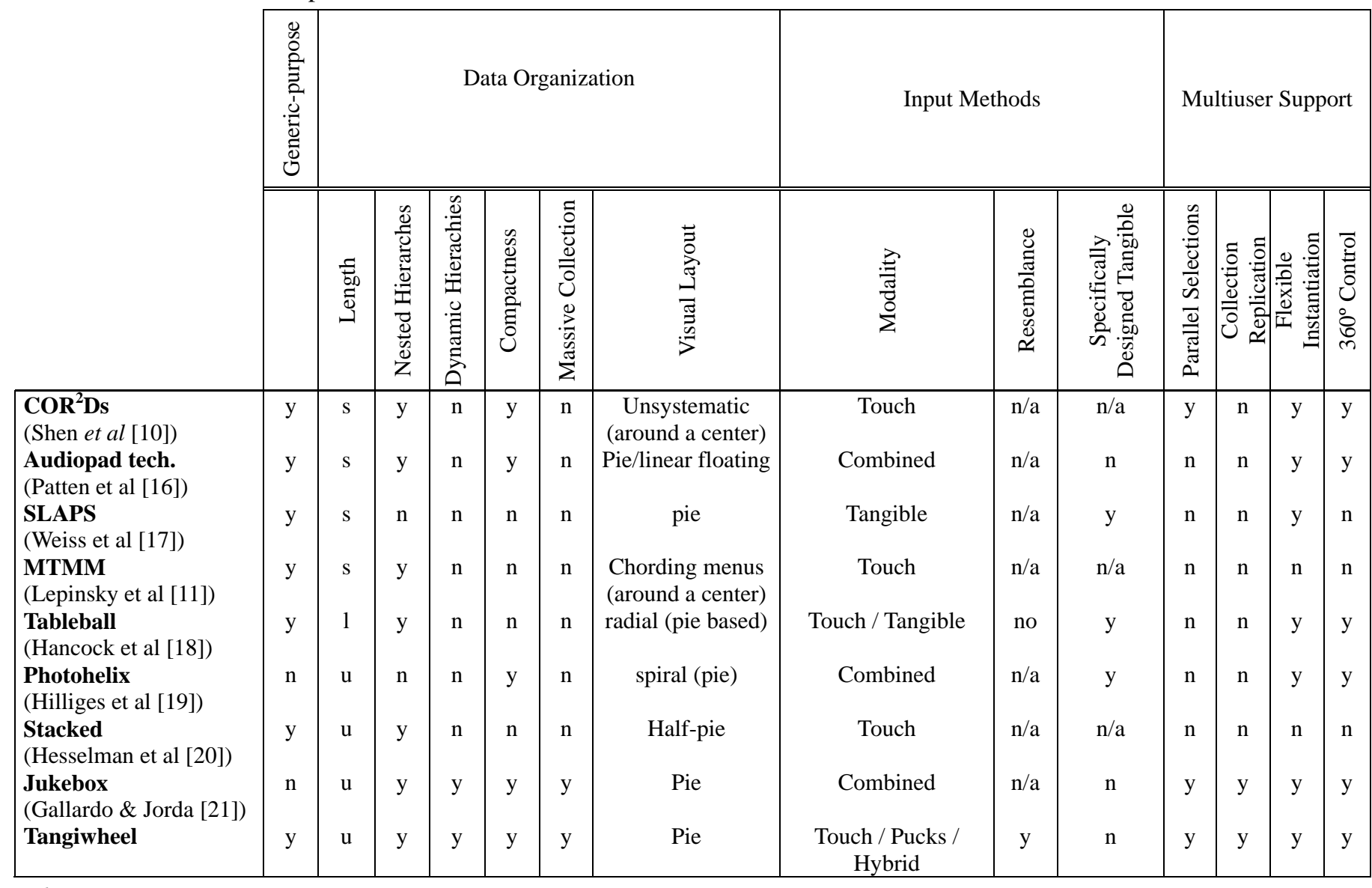


input methods, modality describes the input modality of the techniques. It refers to the supported input modalities: purely multi-touch, purely tangible, combined or flexible hybrid. The difference between the combined and the hybrid input modality is that the former requires an ad hoc combination of modalities (touch and tangible) from the user, according to the actions to be performed with the widget. The latter supports the primary functionality in both modalities and the user selects the most convenient modality for a given input. Some proposals provide a control using a single input modality or even a combination of tangible and touch inputs to support the primary function of the control. Another possibility is to provide dual techniques separately, offering a multi-touch finger-based input and another using tangible. It is then necessary to distinguish the resemblance between these dual input techniques in the homogeneity of the observable behavior of the collection management system with respect to the input modality. To avoid unnecessary cognitive efforts, a high level of resemblance is a desirable feature.

Another important feature for the design of tangible inputs is whether the tangible device used has been specifically designed or whether it is a widely used tangible model (e.g. pucks or cards). This implies easier acceptation and adoption in existing platforms [22].

Another aspect of interest is multi-user support. This set of features determines whether the proposal was designed for multi-user contexts or for single-user scenarios. The $360^{\circ}$ control design reports on whether the proposal being properly seen from different points around the tabletop. In addition to other features already commented in the data organization that can contribute to multi-user support, such as massive collection, parallel selections indicates whether the proposal allows the selection of several items in the same collections in parallel. Collection replication refers to the ability to clone collections to be explored separately by several users or simply create two different views. Flexible instantiation shows whether the proposal supports a flexible mechanism to facilitate accessibility and instantiation of collections.

The comparative analysis of the reported studies reveals that almost all the proposals are designed for generic purpose domains and use a pie-based or similar visual item arrangement system around a center. Nearly half of the proposals provide support for short collections only, and most do not fully consider multiuser contexts. Consequently, they do not facilitate massive comprehensive collection displays, collection replication, or parallel selections. For input modality, the proposals normally rely on a combination of touch and tangible techniques and only one offers two control versions supporting exploration in both input modalities.

Based on the previous analysis, TangiWheel is conceptually designed to overcome the weaknesses of previous systems and provide full support for new features, including collection breadth and depth, as it is desirable to have both virtually 
unlimited length and nested collections. Moreover, the proposal should support massive collection use by multiple users or single-user manipulation of several collections at once. Compactness should also be considered together with other additional features to provide flexibility and ease of use, such as collection replication and $360^{\circ}$ control view. Dual input techniques should be provided, resembling each other as much as possible. It would facilitate the emergence of a hybrid input scheme, instead of a simple combination of input modalities, allowing users to select the best input at manipulation time without any additional cognitive effort. Finally, the tangible device should be standard and widely accepted, instead of a specifically designed system for the application in hand.

\section{TangiWheel: Design Discussion and Imple- mentation}

In this section we present the detailed design and implementation of TangiWheel in terms of the previously introduced aspects and corresponding features.

\subsection{General-Purpose Use}
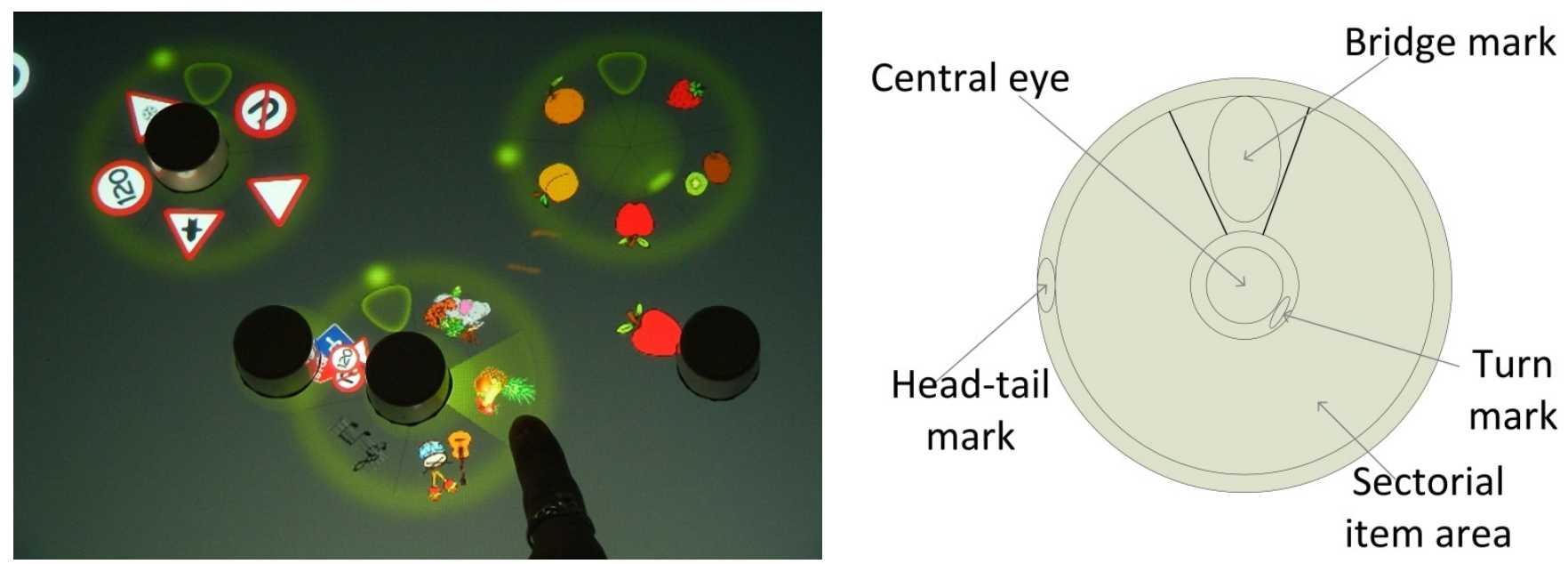

Fig. 1. Right: TangiWheel controls in action; Left: Regions in a TangiWheel instance.
Data intensive applications normally focus on exploring, retrieving information and selecting data of interest. This is the case of applications aiming at constructing artifacts, which require exploring and selecting their component parts. For instance, when creating a technical sketch from existing parts for concurrent exploration and selection on several collections by multiple users, while others assemble the parts using multi-touch input techniques to speed up collaboration. Another possibility is the specification of reactive behavioral rules in simulation environments, requiring the selection of the events, operations and entities involved, possibly ordered by event type, operation type or entity type in nested collections. In the end, we aim to support interaction scenarios on tabletops requiring massive collection exploration, facilitating concurrent collections as a consequence of either several collections that a single user needs or that must be shared, replicated and operated by multiple users. As these scenarios can be diverse, control design should be as flexible, generic and easy-to-use as possible to suit such a wide range of user scenarios and requirements. 
TangiWheel has been successfully deployed to support multi-user creation of articulated entities subject to the rules of physics [23][24] and also to support the collective creation of Rube-Goldberg machines as described in [25] in the context of a much broader research field of creative learning for teenagers on tabletops.

\subsection{Data Organization}

The main design decision of TangiWheel with respect to the data organization aspect was influenced by the need to support conflicting features (see Table 1) such as handling heavily populated collections and sub-collections in a compact way, i.e. without compromising the available tabletop space and thus allowing many instances to be present on the surface.

In this respect, TangiWheel displays the visible items arranged in a circle following the pie menu model. We consider this appropriate, since it is not only compact but also reduces seeking-time and selection errors on visible items by fixing the distance factor [26] and not pre-establishing orientation in a specific direction. An alternative design based on a linear arrangement, which is widely used in other technological platforms (i.e. mostly in desktop based metaphors for single-users) was not considered appropriate, even though it can provide an arrangement that people are more used to, and probably more in line with the way they usually read and consume written information. The fact that TangiWheel is also designed to support (see below) the concurrent manipulation of collections containing both textual and graphical elements in multi-user environments to motivate collective creativity contributed to this decision. Orienting the collection towards a specific user using a linear list would interfere with equal concurrent access to items in collections by multiple users in a tabletop setting. As illustrated in Fig. 1, the control consists of items represented by text or icons, and marked areas for interaction or visualization.

TangiWheel displays only a subset of the elements so that the collection is compact no matter how many elements it contains. This also means that it has the same appearance whatever its length. To explore the invisible elements, TangiWheel has a special region, the bridge mark (see Fig. 1-right), located in a sector of the widget in the form of a button. Simply tapping the button switches between the exploration and rotation modes, which allows for virtually unlimited collections. In exploration mode, rotating the widget entails sliding the visible items in the pie, which means that some items are hidden below the bridge mark while others are shown in their place. To obtain maximum compactness when several collections are on the surface, a minimization state is supported in which all the contained elements are hidden. This state is reached/left by means of prolonged tapping on the bridge mark.

As we have a pie arrangement, we need to keep buttons or any other actionable elements near the center point without breaking the visual circular shape of the widget. Besides, placing the bridge mark in a sector makes the presence of a 
border region naturally represent the place where items are hidden. This design is also flexible, because it offers the possibility of including additional virtual buttons in this special region if it should become necessary to support further functions in the future.

Another visual feedback included in TangiWheel is a brighter point in the perimeter of the pie called head-tail mark (see Fig. 1-right). Since the widget supports collections of unlimited length, the head-tail mark gives users a clue on what is the relative position of the displayed items within the overall collection, similar to the box provided in traditional scrollbars. Although not currently implemented, an additional improvement to this would be to show an expanded preview of several adjacent items near the bridge mark, to further help users in which direction they should explore. Another feature that could improve data visualization when handling very large collections would be the inclusion of a collection of recently-used elements around the bridge mark for direct access. These add-ons will be included in future versions of the widget. Finally, the turn mark provides information on how far the orientation of the control is from the rotation needed to show another invisible element.

The other important feature with respect to data organization is the hierarchical arrangement of collections so that unlimited numbers of nested sub-collections may be defined. Sub-collections may either be statically (at instantiation time) or dynamically created to support the dynamic cou- pling and decoupling of collections with an arbitrary number of nested sub-collections. Hierarchical collections can be created dynamically by simply dragging an existing collection and releasing it into another one. The dragged sub-collection is inserted as an item in the section in which it is released. Once two collections are coupled, their relationship is shown graphically by means of a link (see Fig. 1) when both are displayed, so that there is a visual feedback even when the collections are at a distance from each other on the surface. To decouple two collections the user may break the link by performing a zigzag movement over the link with a puck or a finger.

An expanded sub-collection is represented as a separate TangiWheel instance (see Fig. 1) for several reasons. Firstly, it is an easy method of supporting collection replication and flexible instantiation by means of pucks (i.e., the ability of direct association), which allows any element (sub-collection or single terminal element) to be associated with pucks for direct-access. In TangiWheel a non-associated or empty puck may be placed over an item in a collection at any time. The puck then becomes associated with it and will contain either a single digital object or a TangiWheel sub-collection, depending on whether the item is or is not a leaf node in the collection. To dissociate a container puck, the user has to perform a simple zigzag movement. This mechanism gives direct-access to sub-collections in deeply organized nested structures and reduces seeking time when items have to be re-visited. This association mechanism may also be activated 
by placing an empty puck at the center of a virtual TangiWheel (displayed as a result of finger selections). Representing collections and sub-collections in the same way makes the previous association mechanism homogeneous from the point of view of the actions required from the user (simply placing an empty puck at the center of the ring or central eye).

Secondly, opening nested collections in separate TangiWheel instances keeps the same exploration process through hierarchical collections, and therefore the input methods designed for a root-level collection are the same as for sub-collections. Additionally, in situations with a large number of collections displayed on the surface, users may easily control orientation, location and visibility of the displayed sub-collections.

This gives users flexibility in deciding how the existing sub-collections are displayed on the available space. An alternative design to the one proposed in this work for handling nested hierarchies would be to expand the sub-collection around the selected item to obtain a more compact layout. However this option would compromise the achievement of other features. For instance, input techniques would have to change to support manipulations in each case and would affect the resemblance and consistency between techniques for root-level and nested collections. If compactness was the primary feature to be supported, then a better alternative would be considering in-place versions of the control. In this case the child collection would simply replace the parent, keeping most of the properties of regular collections while being operated in the same way. A future version of TangiWheel will be context-aware and will decide whether to display sub-collections by replacing in-place parent collections (on heavily populated surfaces) or to display them as separate TangiWheel instances, as in the current version of the widget.

\subsection{Input Methods}

In this aspect we consider some of the features related to input management when handling collections and the associated design solutions implemented in TangiWheel to cope with them. Two main features were considered here: modality and resemblance.

TangiWheel supports pure tangible, pure multi-touch or hybrid interaction modes. In tangible mode, a puck is tracked across the surface keeping control rotation and position consistently tethered in rotation mode. In exploration mode, rotating the puck changes the items currently visible. A pie arrangement integrates this interaction more intuitively, since exploring by rotating seems more natural in a circular layout rather than a linear one, in which displacements rather than rotations could be expected. If the puck leaves the surface, the widget disappears but reappears if the puck is repositioned. Items can also be selected by means of tangibles. The use of tangible pucks for selection is based on the idea of tangibles as hypercards, phicons and phandlers [9] for containment and transportation of digital elements. 
In the case of completely virtual TangiWheels (i.e. those created by finger selections on non-leaf items or from specific hotspots), the preceding function is also supported in a multi-touch input modality. Rotation of the widget or exploration of invisible items is performed by a circular movement of the finger following the sectorial item area. An alternative to finger-controlled rotation would be a bi-manual technique using two fingers to describe opposite trajectories, as in [5]. Nevertheless, this technique would affect our resemblance criterion, since the manipulation of a single tangible only requires a single-handed interaction. However, in the adopted input action, in which rotating means exploring, it is very likely when exploring large collections that the rotary action will be continuous and steady to achieve several full $360^{\circ}$ rotations of the collection. Therefore, while describing circles can be performed continuously and without lifting the finger from the surface, the bi-manual technique would require repeatedly re-starting the interaction as soon as both fingers meet. It would make this alternative less effective for data exploration. Finally, in multi-touch modality, the closing action is performed by tapping on the central eye and dragging the widget to a new position on the surface.

\subsection{Multi-user support}

TangiWheel is designed to be used in scenarios in which multiple users may collaborate in the creative process by working together on a common creation space (the surface) and sharing multiple collections of elementary constituent elements used as building blocks to create complex -12 - composite entities. All the team members must therefore have equal access to the collections and a similar perception of them, no matter where they are located around the tabletop. Thus, a 360 -degree control and reach mechanism [27] is a key feature in TangiWheel to make the widget re-orientable and usable by multiple users in a shared space. In addition, to facilitate user access from different points, techniques are provided to move and re-position widgets on the tabletop, unlike other approaches described above, in which either collections or sub-collections are attached to predefined fixed points.

The pie-based arrangement in TangiWheel avoids facing information towards a single fixed point. Nevertheless, if a user prefers a different orientation of the widget with respect to his/her location, he/she may change the widget from exploration to rotation mode, so that rotating entails the rotation of the graphic control: visible items will still be visible but the whole control will face in a new direction. Visible items simply adopt a new position, keeping their location with respect to each other while facing in a new direction. Moreover, some of the mechanisms that were previously discussed are powerful characteristics that facilitate access in multi-user scenarios. These mechanisms are: re-locating the widget at any point on the surface; the association of pucks to items and collections that can be removed and replaced on the surface wherever needed; the support of nested hierarchies and the parallel manipulation of replicated collections. 


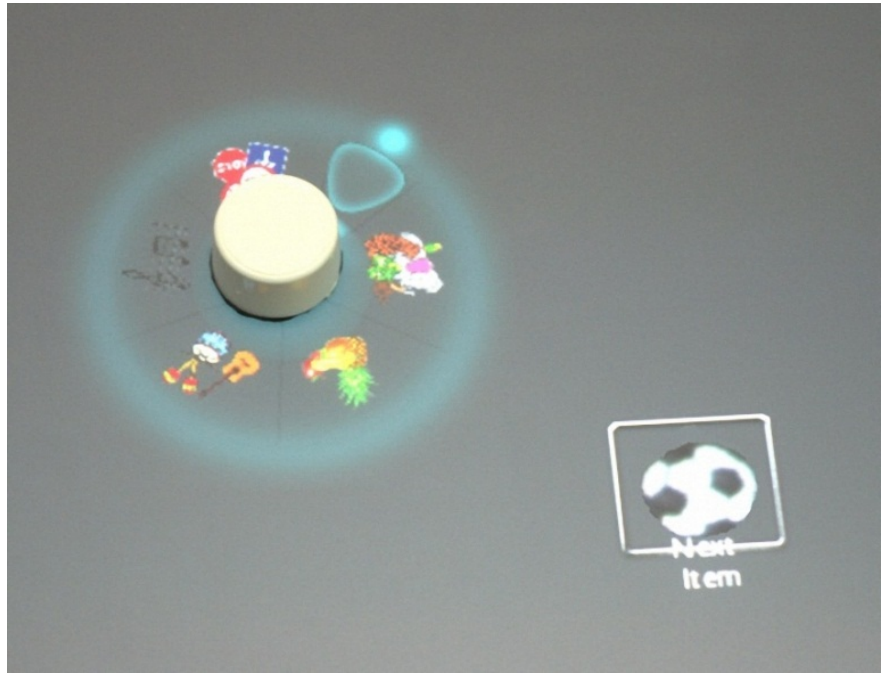

Fig. 2. Interactions 0 and 1 . To select the Soccer ball item (S0), a tangible is first put on the surface (U1) after which a category menu is shown (S1).

\subsection{An example of interaction}

As examples of the proposed techniques in action, Fig. 2, Fig. 3 and Fig. 4 illustrate the interactions required to select an item from a collection and take it to a specific sensitive area on the surface. The performance is explained in terms of user actions $(U x)$ and system responses $(S x)$ as follows:

SO: The application shows a target item to be selected representing a Soccer ball.

$\underline{U 1}$ : User places an empty tangible puck on the surface.

S1: The system deploys a collection of categories implicitly associated with the puck. The control will therefore respond to puck rotations and movements as described above.

$\underline{U 2}$ : As the Sports category is not visible the user needs to explore the collection. To do so he taps on the bridge mark.

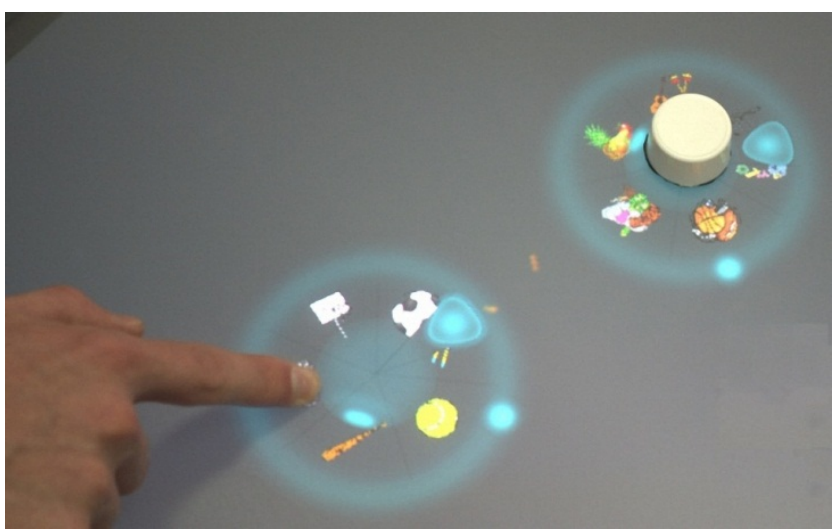

Fig. 3. Interactions 2, 3, and 4. The user has enabled the exploration mode (U2) and has rotated the puck until the Sports category has been displayed and tapped on the item (U3). The user explores the Sport submenu by dragging his/her finger over the items (U4).

S2: The menu toggles to the exploration mode, since it was previously in rotation mode.

U3: The user rotates the puck, changing the visible items on display and taps on Sports when it appears.

S3: In response to this selection, since the item is non-leaf, a completely virtual TangiWheel is displayed to explore the hierarchical sub-collection. In this example the sports collection is displayed, containing items such as tennis ball, bicycle, and so on.

$\underline{U 4}$ : Since this sub-collection has been selected by a finger, it will have to be manipulated by a finger unless a puck is explicitly associated to the widget to turn to tangible interaction. The user can use either method to find the Soccer ball.

S4: The items being displayed change as the user explores the collection.

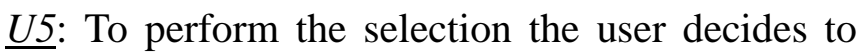



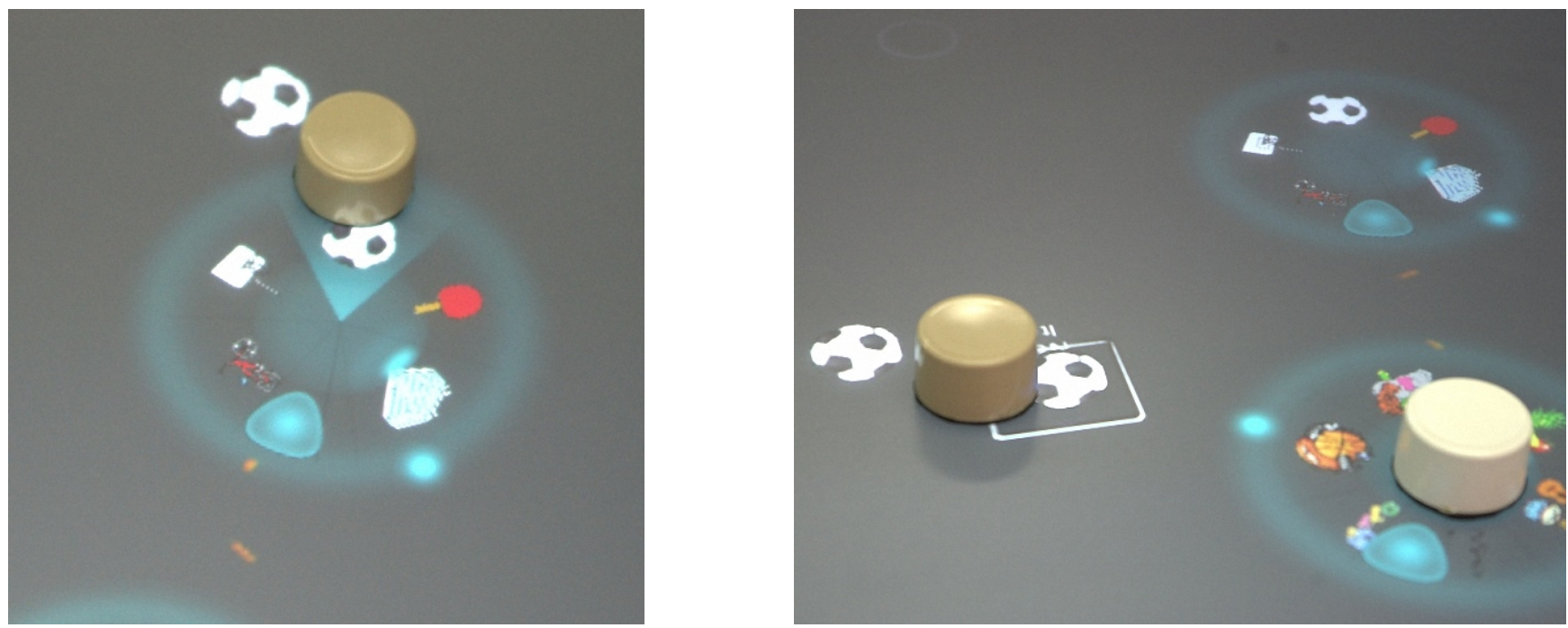

Fig. 4. Interactions 5 (left) and 6 (right). The Soccer ball is selected by using a tangible (U5) and it is then brought to the target area (U6).

use tangibles. He puts a puck on the item.

S5: The item is then virtually linked to this tangible.

U6: The puck containing the Soccer ball item can be placed over the target area to accomplish the goal.

\section{Experimental Context}

Of the different aspects considered in the design of TangiWheel - data organization, input methods and multi-user support - in this paper we describe the experimental evaluation of the techniques used in each input modality. The remaining questions will be dealt with in future research. The following aspects are considered in the present study:

- Acquisition and basic manipulations typically performed to establish the location and orientation of collections. of category collections.

- Series edition (composed of items) that requires not only exploring collections but also insertion or deletion of items.

The overall goal of our experimental design was to study the effect of input method, i.e. fingers or handlers (pucks), on performance in manipulating collections in terms of performance time and number of manipulations required. Experimental complexity was gradually increased to evaluate the use of the widget under different circumstances. TangiWheel is the first tabletop-oriented widget for manipulating collections that supports a hybrid input method with high levels of resemblance. It is therefore important to obtain experimental evidence on what types of situations would benefit from this new type of hybrid input method with respect to pure multi-touch or pure tangible in the context of our proposal. 


\subsection{Participants}

Twenty-three volunteers participated in our study, 16 male and 7 female, mostly undergraduates or Ph.D. students from our university. One participant was left-handed and two were ambidextrous. Ages ranged from 21 to 40 ( $\mathrm{M}=27.5$, $\mathrm{SD}=5.23)$. Eighteen participants reported using a personal computer every day, three almost every day, and two reported using one once or twice per week. Thirteen participants stated they were regular users of touch-enabled devices, whereas ten had seldom or never used one. Eight participants had not had any previous experience of surface computers and the remainder had had limited experience, mostly at shows and exhibitions.

\subsection{Equipment}

The interaction framework for TangiWheel was implemented in C\# using Microsoft Surface SDK v.1.0. with the core libraries specific to Microsoft XNA Framework. The tangibles (i.e. pucks) were tagged with a standard tagbyte set compatible with Microsoft Surface, two units of which were used to implement the experiments.

\subsection{Method}

Test sessions were arranged according to participants' availability. In order to avoid participants learning by observing peer-actions, only two people were in the laboratory at the same time but at two different surface units separated by a folding screen. The participants received an introductory talk, a live demonstration of typical interactions, followed by free, although supervised, interaction training. This procedure took about 15 20 minutes until the participants felt comforta- ble enough with the interaction mechanisms. The study itself began with each participant on his/her own performing the experiments on acquisition and basic manipulations, followed by the selection experiments and the series-edition experiments. In order to avoid all participants interacting with the different alternative designs in the same order, the interaction input modality was established according to a Latin Square design. Participants were encouraged to perform as fast as possible with a reward for the fastest participant.

The task execution-support software automatically recorded all the input interactions in a $\log$ file. The set of logs were post-processed to obtain information for the quantitative statistical analysis. The main information extracted was related to task completion times and the corresponding number of actions required. Different parts of each session were video recorded for further analysis, basically to study participants' behavior patterns. Several questionnaires were also filled in by the volunteers to assess ease of use and effectiveness.

\section{Experiment 1: Acquisition and Basic Ma- nipulation}

At least two interaction phases are involved in the use of an interface element [4], typically, acquisition and manipulation. In the TangiWheel widget, acquisition and basic manipulation tasks are necessary to establish the position and orientation of the control before exploring and selecting an element. The goal of this experiment was therefore to explore the effectiveness of each input method in performing the typical preliminary in- 

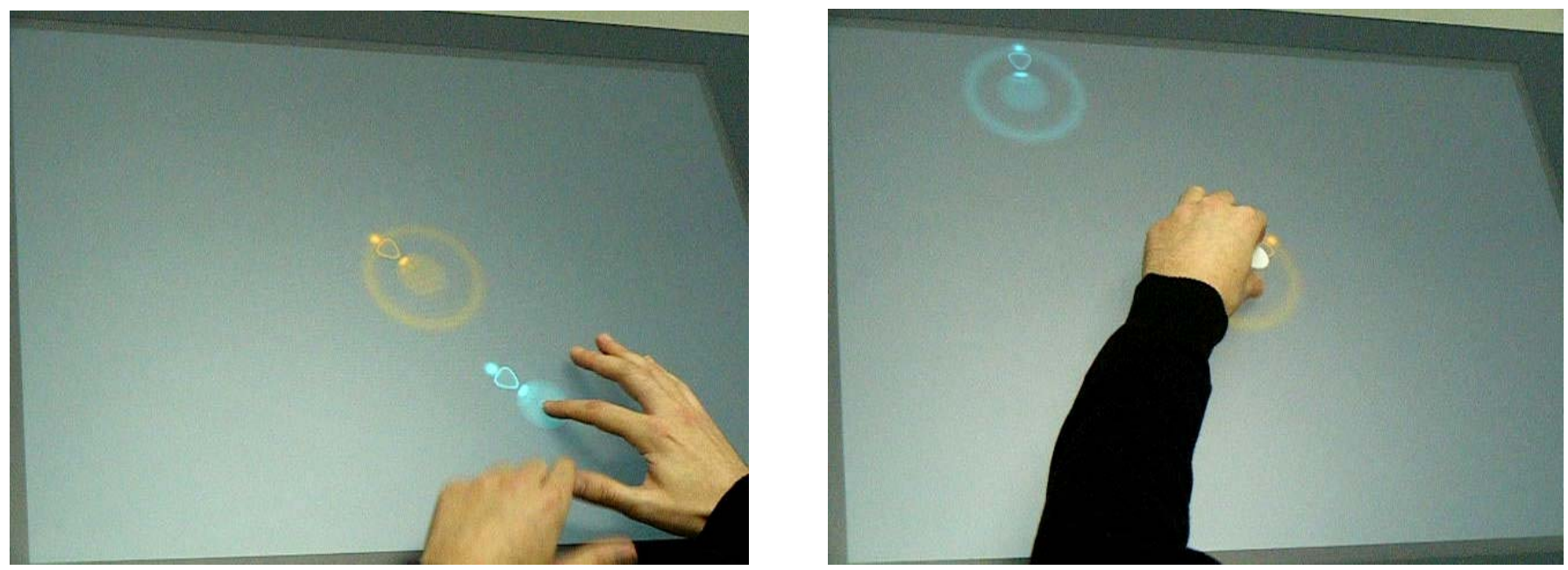

Fig. 5. Interaction in the Acquisition and basic manipulation task (Touch condition on the left, Tangible on the right).

teractions.

\subsection{Task}

Participants are requested to establish the position and orientation of a series of 25 TangiWheel widgets. An orange target with the desired orientation is displayed in the center of the tabletop. A single green TangiWheel widget appears in a predefined pseudo-random position (see Fig. 5). The user has to acquire and perform basic manipulations to match the position and orientation of the widget with the target. When they match, the widget disappears, the target takes on another ori-

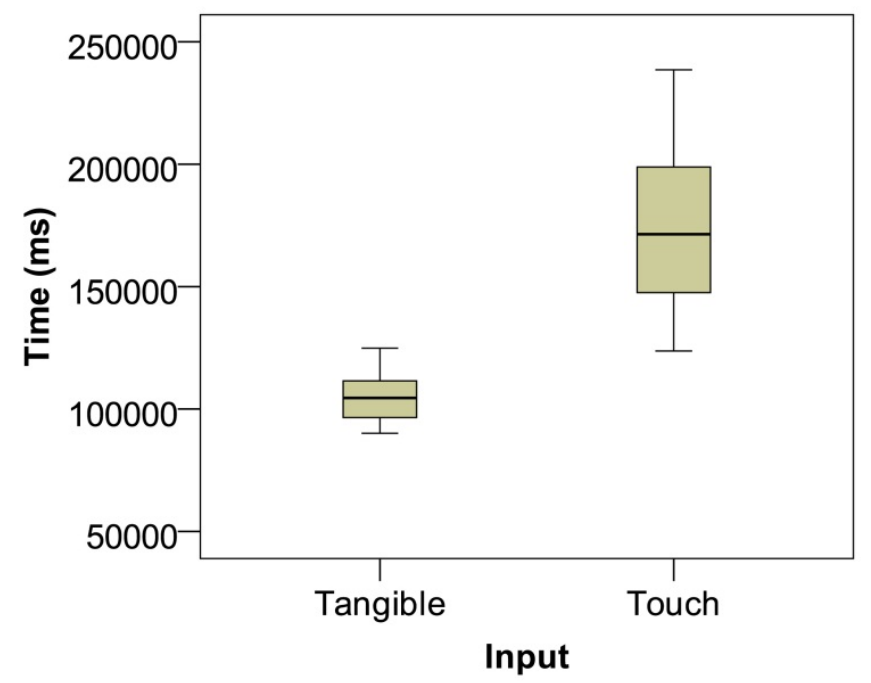

entation and a new widget appears in another location. The series of positions and orientations are predefined but the order of appearance is shuffled for each run.

\subsection{Procedure}

This experiment was performed by each participant twice, once using the tangible input method and once using fingers only. The input method was assigned to each participant according to a Latin Square design in order to avoid order effects. In the Tangible method, the participant had to take the tangible in one hand, acquire the widg-

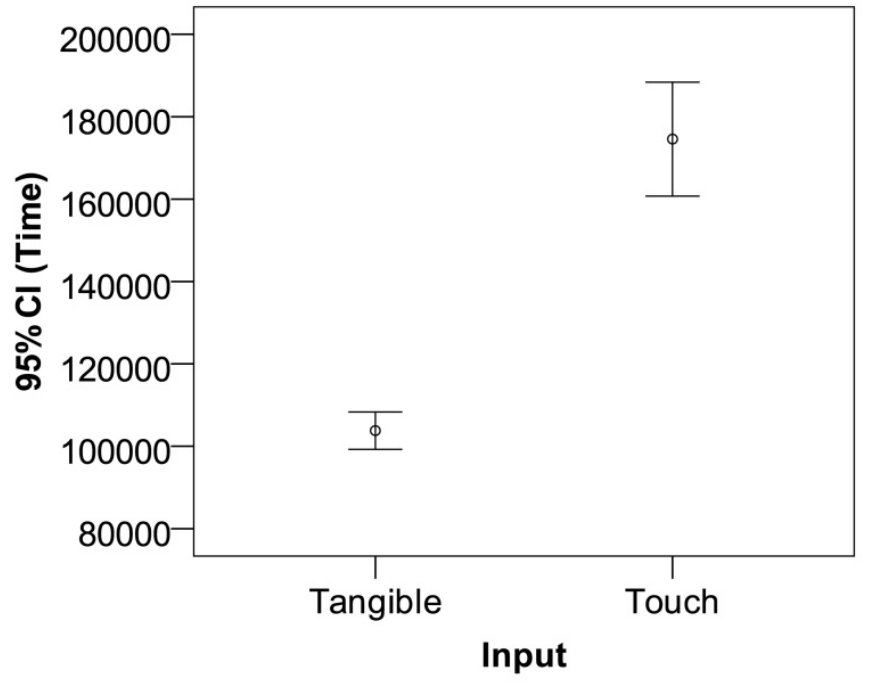

Fig. 6. Box-plot and Confidence Interval plot for $\mathrm{CT}_{\mathrm{BAS}}$. 
et by putting the tangible in its inner region in order to make the binding, and then take it to the target position, performing all the required rotation adjustments. Matching with the target was evaluated when the tangible remained still for about 250ms. In the Touch method, the participant had to accomplish the positioning by dragging the widget from its inner region and adjust orientation by describing circles on the widget with a finger. The evaluation was made when the finger released the widget. The participant had to establish the position of each widget as quickly as possible according to the instructions.

\subsection{Results}

Three variables were measured for each input method: time to complete the experiment $C T_{B A S}$, time to accomplish a single matching $\mathrm{T}_{\mathrm{BAS}}$ and number of actions needed to complete each matching $A_{B A S}$. The superscripts TOUCH and TANGIBLE were used with each variable for notation purposes.

As can be seen in Fig. 6, on average, the participants took longer to complete the task in the Touch condition $C T_{B A S}{ }^{\text {Touch }} \quad(\mathrm{M}=174.56 \mathrm{~s}$, $\mathrm{SD}=31.210$ ) than the Tangible $C T_{B A S}^{\text {Tangi- }}$ ${ }^{\text {ble }}(\mathrm{M}=103.799 \mathrm{~s}, \mathrm{SD}=9.714)$. The analysis of variance (ANOVA) conducted on the time needed to complete the experiment demonstrated that the input method has an effect on the preliminary acquisition and basic manipulations $(\mathrm{F}=$ 94.327, $\mathrm{p}=.000$ ) and that the tangible input outperforms touch. This result agrees with other empirical studies involving repositioning of different interface elements on both multi-touch and tangible interfaces [5][6], which showed that tangible interfaces are effective in spatial layout tasks. Our experiments therefore provide additional empirical evidence on this issue with respect to our techniques for repositioning and reorienting the widget.

In this respect, the result can be explained by the fact that hands are used to rotate and position objects in many everyday actions (grasping) rather than single fingers, whose primary function is to touch (tapping). Grasping objects is therefore a more natural interaction for moving objects than dragging them across a surface with the fingers, considering the techniques involved. Moreover, the Touch condition suffered from an interaction issue already observed and reported in [5] as exit error. This refers to the difficulty of disengaging from the virtual object without causing some form of unintended extra movement. This is an inherent problem in touch input on tabletops that may affect users to different degrees. It was seen to affect the correct positioning of widgets and in the end contributed to considerably higher times in the Touch condition.

A study was made of the time needed to take the widget to each target and the number of actions required. Matching single targets took more time with Touch $T_{B A S}{ }^{\text {Touch }}(\mathrm{M}=5.861 \mathrm{~s}, \mathrm{SD}=2.024)$ than Tangible $T_{B A S}$ Tangible $(\mathrm{M}=3.772 \mathrm{~s}, \quad \mathrm{SD}=0.930)$. However, fewer actions were required using Touch $A_{B A S}{ }^{\text {Touch }}(\mathrm{M}=3.45, \mathrm{SD}=2.53)$ than Tangible $A_{B A S-}$ Tangible $(\mathrm{M}=7.17, \mathrm{SD}=2.43)$. This can be explained by the fact that a complete rotation of the widget 
can be performed continuously with a finger describing circles on the surface, which counts as a single action. However, when the same action is performed by a puck, the continuous rotation cannot be reproduced, so that every movement of the hand counts as an additional action.

Since the assumption of normality was not met, a comparison was made using Mann-Whitney tests. Significant differences were found in the time taken to accomplish a single target $\left(\mathrm{H}_{0}: T_{B A S-}\right.$ Tangible $=T_{B A S}{ }^{\text {Touch }} ; \mathrm{z}=-19.06$, $\mathrm{p}$-value $\left.=.000\right)$ and also in the number of actions required $\left(\mathrm{H}_{0}\right.$ : $A_{B A S}{ }^{\text {Tangi- }}$ ble $=A_{B A S}{ }^{\text {Touch }} ; \mathrm{z}=-22.46$, $\mathrm{p}$-value= $\left.=000\right)$.

In view of these results, although it could be thought that our finger-touch mechanism is not a suitable interaction technique for TangiWheel, this premature conclusion will be questioned in Experiment 2.
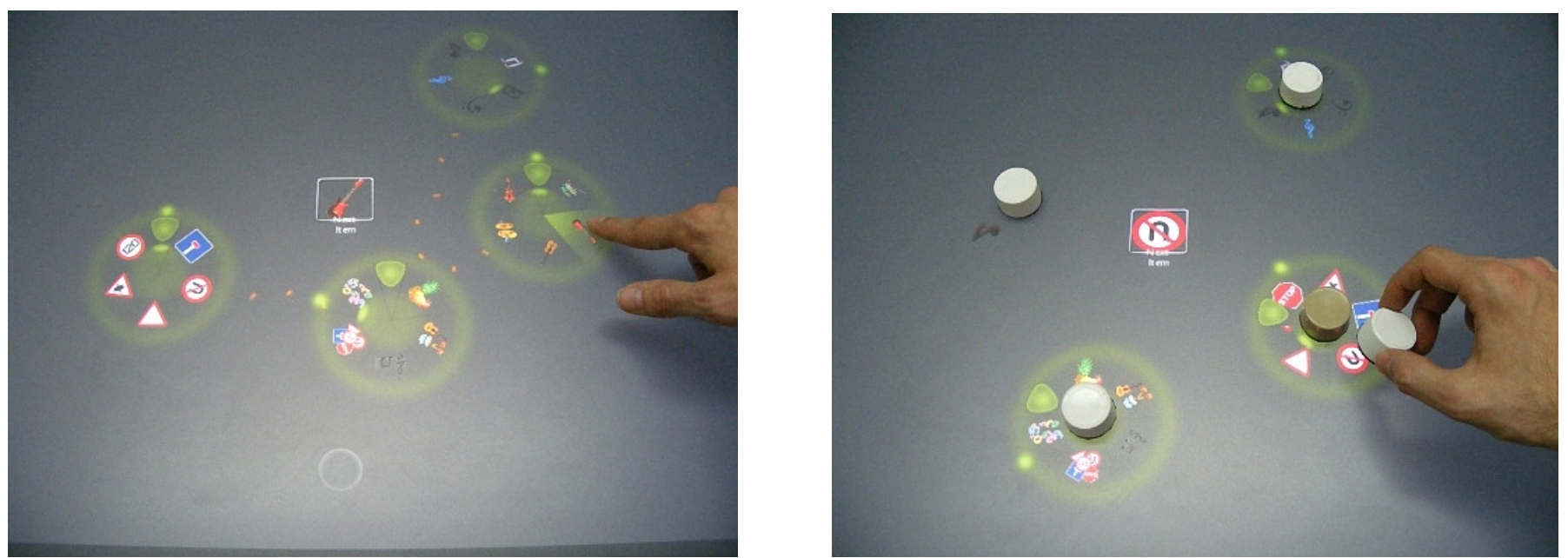

Fig. 7. Interaction in the MTS task (Touch on the left, Tangible on the right). 

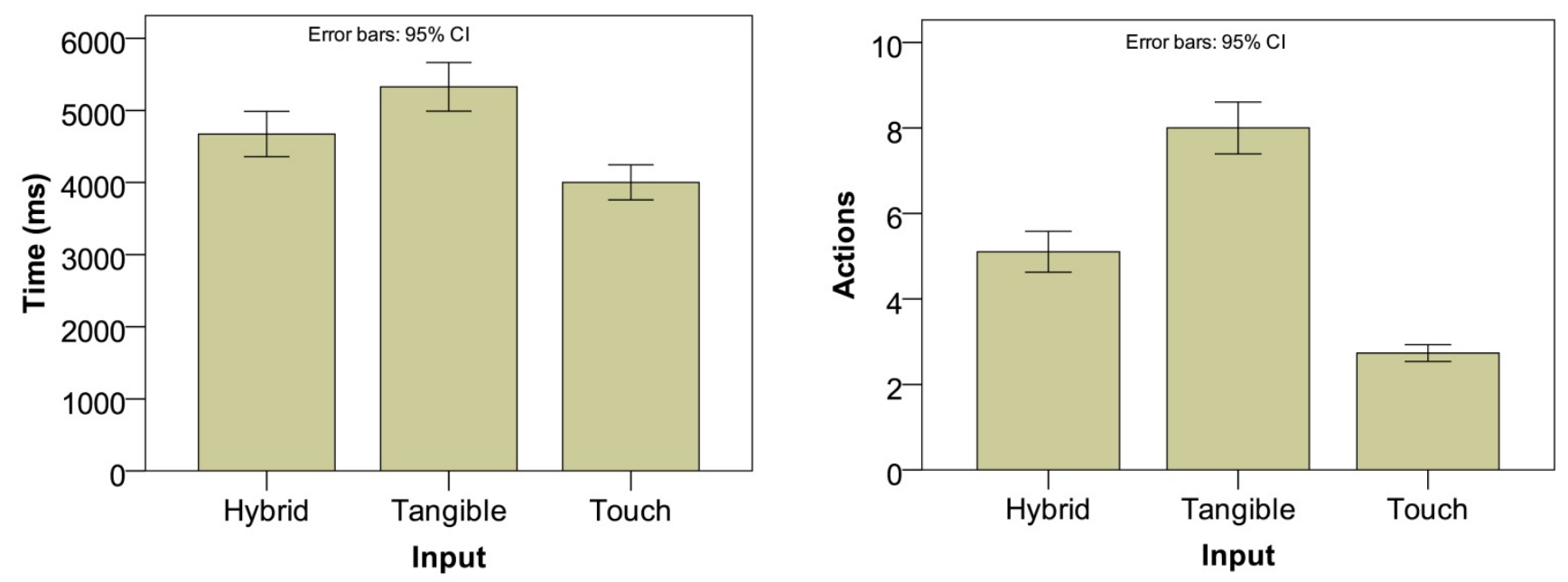

Fig. 8. Mean plots for times $T_{M T S}$ (left) and number of actions $A_{M T S}$ (right).

\subsection{Procedure}

Each participant performed three runs to vary the input method: touch, tangible and hybrid. The order of the runs was arranged according to a Latin Square design to avoid order effects. While the hybrid input method allows the use of pucks and fingers in any combination, following the full functionality of the TangiWheel widget, the tangitangible and touch conditions only responded to pucks or fingers, respectively. This means that in the tangible condition the pucks are used both to open collections and to select items whereas in the touch condition explorations and selections are made with fingers only (see Fig. 7).

\subsection{Results}

The application of ANOVA to the experimental times demonstrated that they are

Table 2. Post-hoc pairwise comparisons using t-tests (Bonferroni corrected) for time required to complete the MTS task.

\begin{tabular}{lll}
\hline \hline Comparison & Mean differences & p-value \\
\hline Hybrid - Tangible & -19662.57 & .013 \\
Hybrid - Touch & 16804.16 & .041 \\
Tangible - Touch & 36466.73 & .000 \\
\hline \hline
\end{tabular}

significantly influenced by input method ( $F=$ 15.512, $\mathrm{p}=.000$ ). Post-hoc pairwise comparisons using t-tests (Bonferroni corrected) showed that all three conditions were significantly different (see Table 2)¡Error! No se encuentra el origen de la referencia.. The results show that the Touch input condition performed best, followed by the Hybrid and Tangible.

We also analyzed the time needed to complete a single target item selection and the number of actions required. Fig. 8-left shows the mean times for each condition. Using fingers only gave better results than the other two input conditions, which are enabled to use pucks. Fig. 8-right shows that the Touch condition also required the lowest number of actions, followed by Hybrid and Tangible. 
As the normality assumption was not met, a pairwise comparison was conducted on the time to complete a single target selection and the number of actions needed using Mann-Whitney tests to determine whether the differences were significant. As can be seen in Table 3, the tests showed there were significant differences between all three input conditions for the two measured variables.

The results indicate that the number of actions and the average time needed to search and select an individual element are also significantly lower for the Touch condition and therefore suggest that fingers are more effective in scenarios requiring focused selections from a collection at a fixed position with no basic manipulations (rotations and translations) on the surface. This technique would therefore be suitable for single users working on interfaces with collections displayed at predefined fixed locations in the work space and a predefined 2D orientation of the interface.

However, in applications in which different subjects have to acquire and bring low-item collections to their personal space, the tangible input method would outperform touch because the number of acquisition actions clearly exceeds the number of explorations in this case. Finally, in scenarios with a relatively high number of acquisi-

Table 3. Mann-Whitney tests to compare $T_{M T S}$ and $A_{M T S}$.

\begin{tabular}{lll}
\hline \hline $\mathrm{H}_{0}$ & $\mathrm{z}$ & $\mathrm{P}$ \\
\hline $\mathrm{T}_{\text {MTS }}{ }_{\text {Hybrid }}=\mathrm{T}_{\text {MTS }}{ }_{\text {Tangible }}$ & -3.089 & .002 \\
$\mathrm{~A}_{\text {MTS }}{ }_{\text {Hybrid }}=\mathrm{A}_{\text {MTS }}$ Tangible & -9.488 & .000 \\
$\mathrm{~T}_{\text {MTS }}{ }_{\text {Hyrid }}=\mathrm{T}_{\text {MTS }}{ }_{\text {Touch }}$ & -2.388 & .017 \\
$\mathrm{~A}_{\text {MTS }}{ }_{\text {Tybrid }}=\mathrm{A}_{\text {MTS }}{ }_{\text {Touch }}$ & -5.932 & .000 \\
$\mathrm{~T}_{\text {MTS }}$ Tangible $=\mathrm{T}_{\text {MTS }}$ Touch & -5.588 & .000 \\
$\mathrm{~A}_{\text {MTS }}{ }^{\text {Tangible }}=\mathrm{A}_{\text {MTS }}{ }^{\text {Touch }}$ & -16.882 & .000 \\
\hline
\end{tabular}

Table 4. Use of fingers/pucks in the hybrid input condition on MTS task.

\begin{tabular}{lllll}
\hline \hline & Finger & \multicolumn{3}{l}{ Puck } \\
\hline Action & Avg. & $\%$ & Avg. & $\%$ \\
\hline Manipulation & 41.60 & 72.5 & 15.78 & 27.5 \\
Menu instantiation & 4.04 & 38.75 & 6.39 & 61.25 \\
Selection & 25.86 & 82.75 & 5.39 & 17.24 \\
Target establish. & 21.30 & 97.23 & 0.61 & 2.77 \\
\hline \hline
\end{tabular}

tion actions and large collections, a hybrid approach would integrate the best features of both interaction modalities. Tangiwheel supports all three modes and, unlike those described above, can therefore be considered a flexible widget that can be effectively used in a wide range of scenarios.

\subsection{Hybrid Performance Results}

Regarding the performance profile on the Hybrid input, Table 4 summarizes the use of fingers and pucks for several important actions in terms of average number of actions per user and overall percentage. The results show that the subjects generally preferred fingers (72.5\%) to pucks (27.5\%), when given the choice. Fingers were mostly used to select and establish the target (82.75\% and $97.23 \%$ respectively). Evidence was obtained from the video recording that subjects had difficulties when using a puck to select an item on a TangiWheel widget.

However, the results also show that the subjects made greater use of pucks to create menus (61.25\%) that were then explored tangibly. This suggests that they found it easier to explore. On average, each participant used almost 6 different pucks $(M=5.7)$ to complete the task, suggesting that they made extensive use of them and did not 


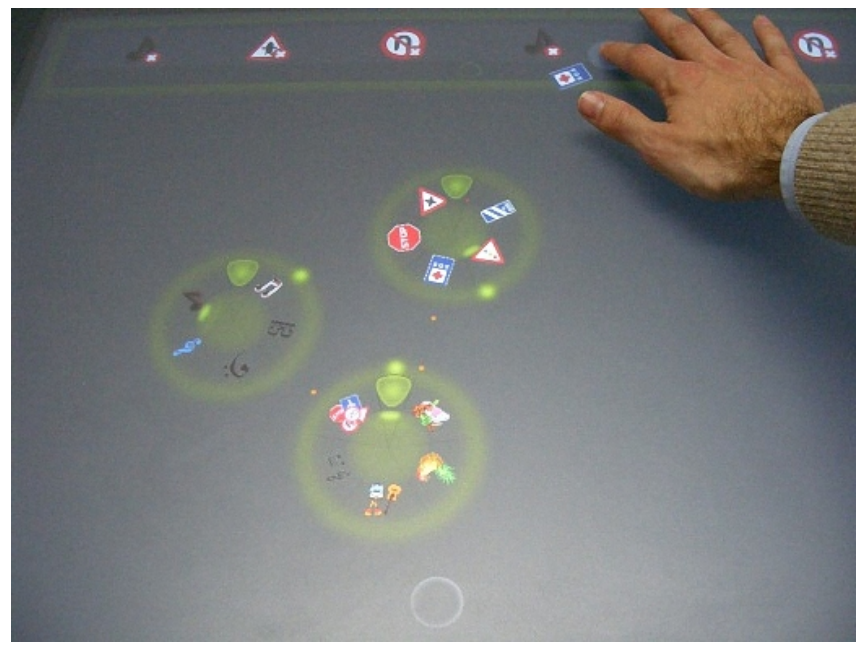

Fig. 9. Edition of series in Touch (on the left) and in Tangible conditions (on the right).

limit the number of pucks used simultaneously.

\section{Experiment 3: Series Edition}

Experiments 1 and 2 evaluated performance in basic manipulation activities under different interaction conditions and the location and selection of elements in structured collections to match individual samples drawn from a series. In other words, there was a one-to-one relationship between the visual stimulus and the target element being manipulated when performing a simple action (rotation, translation and selection). However, to fully explore the possible influence on performance of the different TangiWheel input conditions, an experiment was designed with a higher number of samples and possible actions. Our main goal was to increase the cognitive overload when manipulating a collection under different conditions and to involve the user in a complex task that requires the combination of rotation, translation, search, selection, insertion and deletion of elements in collections. This would allow us to simulate an authentic user scenario by increasing cognitive complexity while maintaining a controlled experimental environment in which a comparative study can be made of the different input modali-

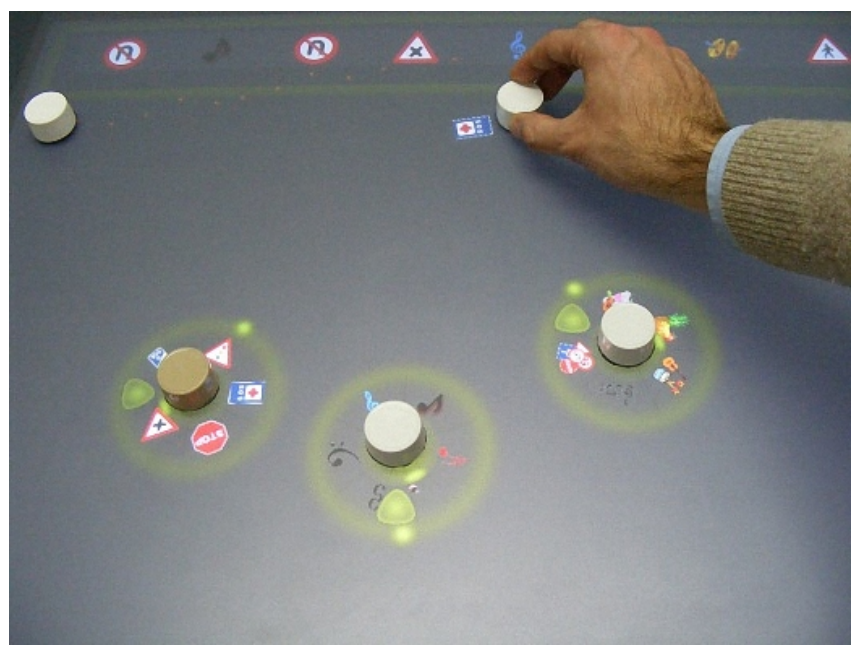

ties.

\subsection{Task}

Participants were requested to either create a series defined as a sequence of elements belonging to different collections, or to modify an existing one. For this, they had to explore existing collections and add or remove elements to/from the series.

\subsection{Procedure}

Three runs of this experiment (Tangible, Touch and Hybrid) were conducted (see Fig. 9). A Latin Square design was used to avoid order effects. Participants were individually given an instruction sheet and were told to create two new series and modify an existing one. The proposed series for each interaction style were of similar difficulty in terms of length and variability of the items included. In the Touch condition the subjects had to search for and select items in TangiWheel collections using their fingers on virtual TangiWheel widgets, which may be rotated or dragged across the surface by using touch, as in the previous experiments. To insert an element in a specific position of the reference series they had to 
Table 5. Comparisons for the time required to complete the series task.

\begin{tabular}{lll}
\hline \hline Comparison & Mean differences & p-value \\
\hline Hybrid - Tangible & -11892.487 & 1.000 \\
Hybrid - Touch & -44728.876 & .018 \\
Tangible - Touch & -32836.389 & .103 \\
\hline \hline
\end{tabular}

drag the element from its collection and drop it in the appropriate position in the sequence, without raising their finger from the surface. To remove an element from a series a deletion button is displayed next to each item. On the other hand, to add an element to the series in the Tangible condition the subjects had to use pucks to search and select items. Since the elements were arranged in several nested categories, they could at any time associate a puck with a category for direct access and use the puck to rotate or move the collection to any area on the surface. Elements were selected by placing a puck over them and were inserted in the proper position in the series by releasing the puck in the desired area. To remove an element from a series, a deletion puck was placed on top of the element.

\subsection{Results}

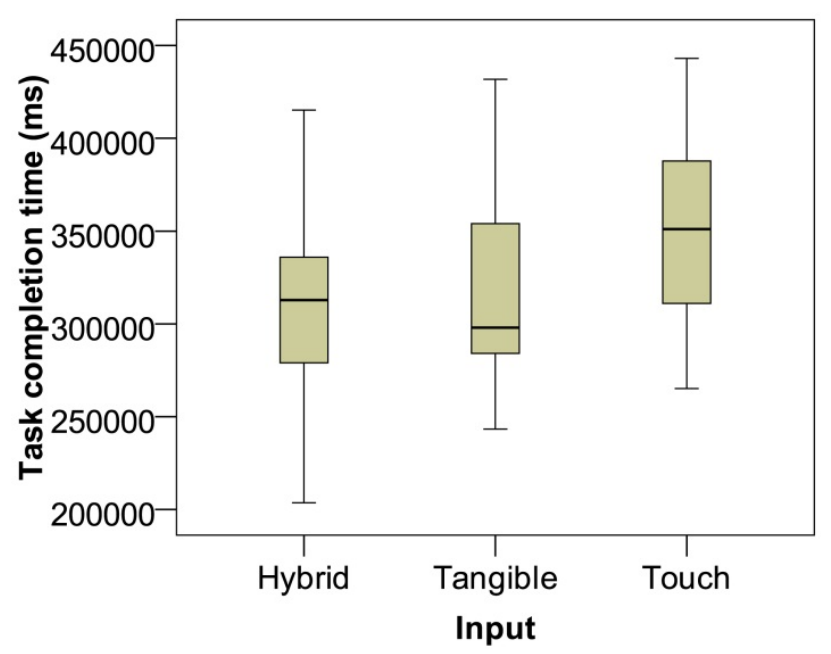

Fig. 10. Box-plots for $C T_{S E R}$ and $T_{S E R}$.
Fig. 10 shows the distribution of the time required to complete the experiment in each condition and the times needed to complete the edition of a single series. The application of ANOVA to the times needed to complete the experiment demonstrated that this is affected by the input method $(\mathrm{F}=4.413, \mathrm{p}=0.016)$.

Post-hoc pairwise comparisons using t-tests (Bonferroni-corrected) showed that Touch is significantly slower than Hybrid, but found no significant differences between Touch and Tangible or between Tangible and Hybrid (see Table 5). Nevertheless, the mean time needed to complete the task was higher for Touch and Tangible than Hybrid, and Touch higher than Tangible.

We also analyzed the time needed to complete the edition of a single series and the number of actions it required. Fig. 11 shows the corresponding mean plots. The application of ANOVA to the time needed to complete a single edition demonstrated that this is affected by the input

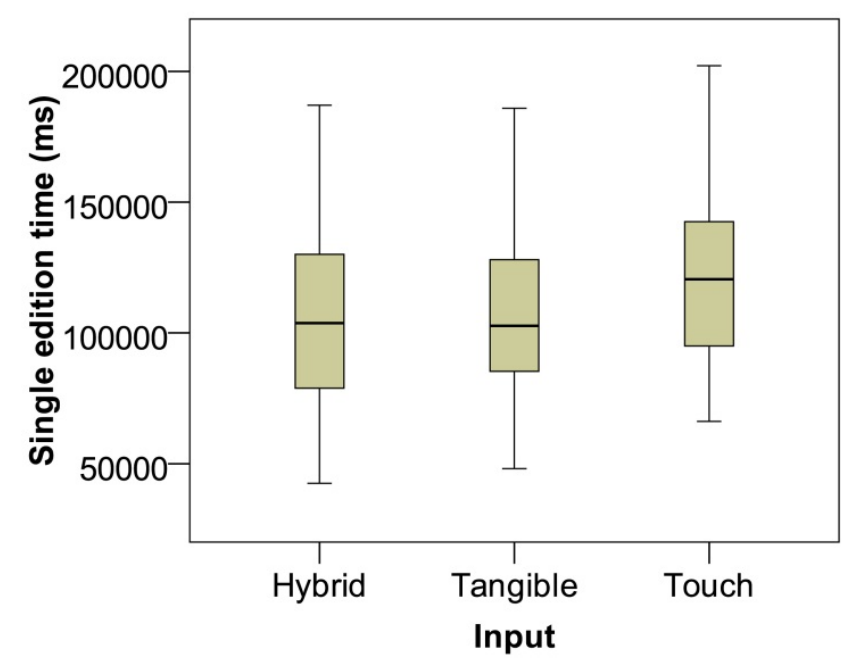



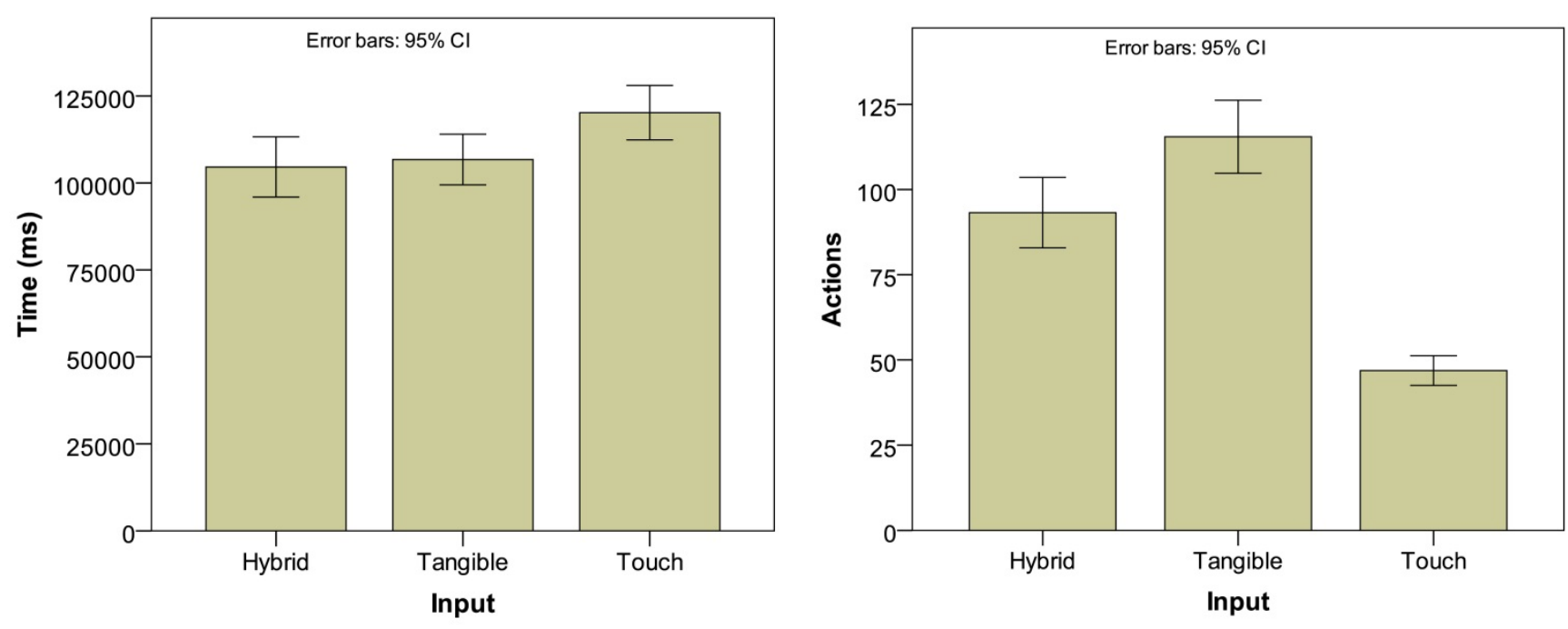

Fig. 11. Mean plots for $T_{S E R}$ (left) and $A_{S E R}$ (right). method $(\mathrm{F}=4.503, \mathrm{p}=.012)$.

Post-hoc pairwise comparisons using t-tests (Bonferroni-corrected) showed that Touch is significantly slower than Hybrid. Touch also fared worse in the comparison with Tangible and is close to significance ( $\mathrm{p}=0.053$ at $5 \% \mathrm{CL}$ ). The tests showed no significant difference between Hybrid and Tangible (see Table 6).

The application of ANOVA to the number of actions required showed that this parameter is affected by the input method ( $\mathrm{F}=60.269, \mathrm{p}=.000)$. Post-hoc pairwise comparisons using t-tests (Bonferroni-corrected) showed that all three conditions were significantly different (see Table 7). Touch required a significantly lower number of actions than Tangible and Hybrid conditions. In addition, Hybrid required a significantly greater number of actions than Tangible.

\begin{tabular}{lll}
\hline \hline Comparison & Mean differences & p-value \\
\hline Hybrid - Tangible & -2153.072 & 1.000 \\
Hybrid - Touch & -15611.345 & .018 \\
Tangible - Touch & -13458.273 & .053 \\
\hline \hline
\end{tabular}

Table 7. Comparisons for $\mathrm{A}_{\mathrm{SER}}$.

\begin{tabular}{lll}
\hline \hline Comparison & Mean differences & p-value \\
\hline Hybrid - Tangible & -22.253 & .002 \\
Hybrid - Touch & 46.350 & .000 \\
Tangible - Touch & 68.603 & .000 \\
\hline \hline
\end{tabular}
vides more effective task completion in terms of the time required than the other two approaches, especially when comparing Hybrid with Touch.

This means that the Hybrid interaction is the most effective for more complex tasks than the one described in the MTS Experiment. This is due to the fact that editing a series requires a combination of the actions studied in the two previous experiments. When this situation arises, the poor performance of the Touch interaction style for basic manipulation tasks (dragging and rotating) makes this approach less effective, even though it shows good behavior for searching and selecting items in collections. In fact, the analysis of the recorded videos reveals two important situations in this respect. Firstly, in both the Hybrid and Tangible conditions the subjects benefited from using

Tangible - Touch $\quad 68.603$ 
pucks as element containers and consistently used a single container puck for multiple insertions of elements that appeared repeatedly in a series. On the other hand, those using the Touch condition had to drag the element across the surface several times to insert it in different parts of the list. This penalizes the task completion time because, as shown in the first experiment, the Touch condition is less effective for basic manipulation activities. Secondly, we also observed that, because the number of elements in a series was high, the number of TangiWheel instances displayed on the surface increased over time, forcing those using Touch to relocate them so that they would not interfere with the task. Again, the need to relocate the TangiWheel instances by touch makes the overall task less effective.

We may conclude from the results obtained that hybrid designs allowing both Tangible and Touch interaction styles outperform those based on touch or tangible-only styles in situations requiring: movement of collections on surfaces that have a great number of elements displayed simultaneously; the re-use of elements in collections in different areas on the surface; and re-orientation of the containers that hold the collections. These requirements are certainly present when several participants share the working space or in situations that require flexible collection layouts. The better performance of the Hybrid interaction style in these situations is important, given that surfaces are mainly designed to support touch-based interactions.
Table 8. Use of fingers/pucks in the hybrid input condition on series task.

\begin{tabular}{lllll}
\hline \hline & Finger & Puck \\
\hline Action & Avg. & $\%$ & Avg. & $\%$ \\
\hline Manipulation & 78.26 & 51.18 & 74.65 & 48.82 \\
Menu instantiation & 8.52 & 61.44 & 5.35 & 38.56 \\
Selection & 9.87 & 30.80 & 22.17 & 69.20 \\
Item insertion & 5.22 & 13.82 & 32.52 & 86.18 \\
\hline \hline
\end{tabular}

\subsubsection{Hybrid Performance Results}

As shown in Table 8,Table the results of the Hybrid input condition showed that pucks were used almost to the same extent as fingers. In contrast to the MTS task results, selection operations with pucks (69.2\%) and menu instantiation using fingers (61.44\%) were preferred. In addition, items were inserted in the series mostly by tangibles. On average, users used 6-7 different pucks to complete the task $(M=6.48)$.

All these results confirm the evidence obtained from the videos. Tangible techniques perform better with increased task difficulty and overload. As the surface easily becomes cluttered and previously selected and inserted items are likely to be reused several times, pucks provide advantages as they are easier to handle in these situations. The subjects appeared to get overworked as the task progressed when dragging items repeatedly with the fingers.

\section{Questionnaire Results}

After the participants had finished each task, they were asked to complete questionnaires on ease of use and usefulness. The 5-point Likert scale questions regarding selection and series task 


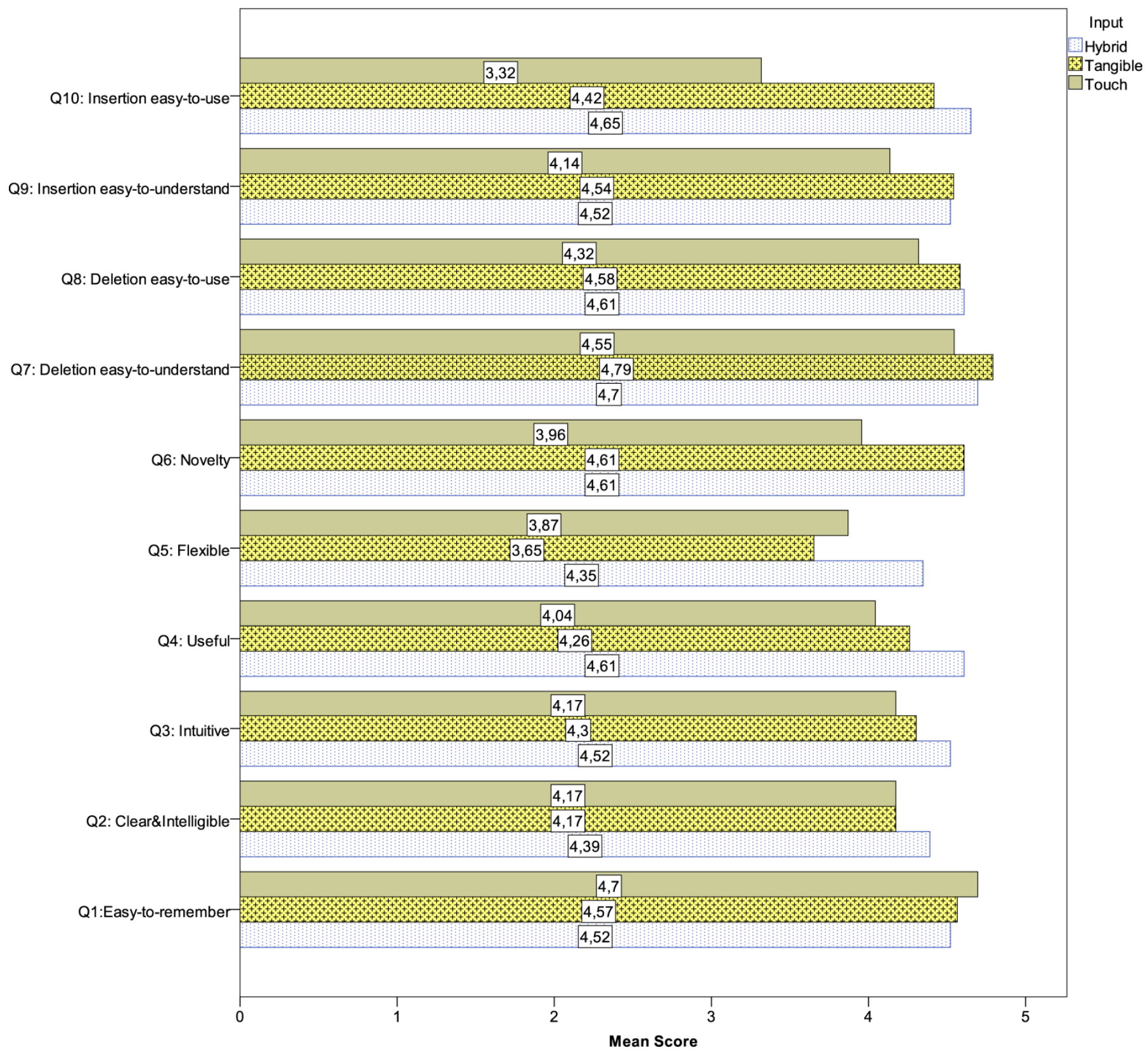

Fig. 12. Mean plots for the basic questionnaire.

were designed to compare the three input methods evaluated by pairwise Mann-Whitney tests. Table

Table 9. Statements scored by participants in the basic questionnaire.

\begin{tabular}{ll}
\hline \hline Q & Questions: I consider that... \\
\hline Q1 & the mechanisms to explore collections are easy to remember. \\
Q2 & the interaction is clear and intelligible. \\
Q3 & the control for collections used is intuitive when handling. \\
Q4 & the mechanism to interact with collections is useful. \\
Q5 & the mechanism to interact with collections is flexible. \\
Q6 & the way of interacting with collections is novel. \\
Q7 & the metaphor for deletion of items is easy to understand. \\
Q8 & the metaphor for deletion of items is easy to use. \\
Q9 & the metaphor for insertion of items is easy to understand. \\
Q10 & the metaphor for insertion of items is easy to use.
\end{tabular}

9 contains the statements that were scored by those who participated in the tests to questions in the basic questionnaire. Fig. 12 shows the mean plots of the average scores.

In general, the three methods were assessed positively and no design issues were raised from the answers. For instance, the subjects found the collection exploration techniques easy to remember in all three methods (Q1), with no significant differences between methods. They also consid- 
ered that the exploration interaction techniques were clear and intelligible (Q2). They found hybrid control techniques to be the most intuitive (Q3). These answers showed that the subjects anticipated the behavior of tangibles when performing natural actions, such as acquisition, rotation and translation, whereas in Touch more complex actions were necessary and therefore required a higher cognitive effort. They considered the hybrid design enabled them to select the most intuitive interactions from the tangible and multi-touch approaches.

Interacting with collections was found to be mostly useful in the three conditions (Q4). However, Hybrid was given a significantly higher score $(\mathrm{z}=-2.896, \mathrm{p}=0.004)$. This result suggest that users perceive as most useful the possibility of selecting the most appropriate input condition according to the task at hand.

There was some discrepancy on method flexibility and novelty. Hybrid was considered more flexible and Tangible more cumbersome (Q5). The differences between Hybrid and Tangible were highly significant $(\mathrm{z}=-2.625, \mathrm{p}=0.009)$ while between Hybrid and Touch they were close to significance $(\mathrm{z}=-1.839, \mathrm{p}=0.066)$. These results suggest that the proposed combination of touch and tangible styles is an advance in flexibility.

They also considered both Hybrid and Tangible to be equally novel (Q6). Touch was thought to be significantly less novel ( $\mathrm{z}=-2.410, \mathrm{p}=0.016)$, which means tangible components increase the perception of novelty.

Questions Q7-Q10 in Fig. 12 depict the mean scores for questions related to the metaphors for inserting and deleting elements. While the metaphors and techniques involved in deletions were considered easy to understand and use in all the input conditions (Q7 and Q8), significant differences were found to exist in insertions. The insertion metaphor in Touch is significantly harder to understand than Tangible according to the test on Q9 scores $(\mathrm{z}=-2.034, \mathrm{p}=0.042)$. Also, the insertion operation in Touch mode is significantly more difficult to handle (Q10) than that in Hybrid $(\mathrm{z}=-4.333, \mathrm{p}=0.000)$ and Tangible $(\mathrm{z}=-3.675$, $\mathrm{p}=0.000$ ) modes. The metaphor associated with obtaining, lifting and releasing a tangible container to insert an item at a specific section within a collection was better understood than having to drag items across the surface with a finger.

Some additional questions were specific to

Table 10. Summary on agreement with specific statements in the Hybrid condition. Participant counts and percentages for each agreement level and question are summarized.

\begin{tabular}{|c|c|c|c|c|c|c|}
\hline$\overline{\mathrm{Q}}$ & Q Questions & $\begin{array}{l}\text { Strongly } \\
\text { disagree }\end{array}$ & Disagree & Neutral & Agree & $\begin{array}{l}\text { Strongly } \\
\text { Agree }\end{array}$ \\
\hline$\overline{\mathrm{HS} 1}$ & Tangibles being able to contain items is advantageous & 0 & $2 / 8.7 \%$ & $3 / 13 \%$ & $8 / 34.8 \%$ & $10 / 43.5 \%$ \\
\hline HS2 & I prefer using virtual components when possible & 0 & $5 / 21.7 \%$ & $13 / 56.5 \%$ & $5 / 21.7 \%$ & 0 \\
\hline HS3 & I prefer using tangibles when possible & 0 & $6 / 26.1 \%$ & $8 / 34.8 \%$ & $8 / 34.8 \%$ & $1 / 4.3 \%$ \\
\hline HS4 & $\begin{array}{l}\text { Using TangiWheel in Hybrid modality improves my effectiveness in } \\
\text { selecting items }\end{array}$ & 0 & $1 / 4.3 \%$ & $2 / 8.7 \%$ & $6 / 26.1 \%$ & $14 / 60.9 \%$ \\
\hline HS5 & $\begin{array}{l}\text { Using TangiWheel in Hybrid modality improves allows me selecting } \\
\text { items quickier }\end{array}$ & 0 & $1 / 4.3 \%$ & 0 & $8 / 34.8 \%$ & $14 / 60.9 \%$ \\
\hline
\end{tabular}


Hybrid selection (HS) in order to assess preferences and perceptions. Table 10Table shows the statements assessed and summarizes to what extent subjects agreed with the statements. The results in general are in favor of the hybrid method. Firstly, the capability of tangibles to contain items was rated positively. Secondly, they were slightly in favor of using tangibles when asked about personal preferences. Thirdly, they felt that using TangiWheel in hybrid mode clearly improves both effectiveness and productivity when selecting items.

At the end of the session, a final questionnaire (FQ) was administered consisting of single-choice questions which explicitly asked which input condition, if any, was perceived as most appropriate in performing specific actions.

As results show (see FQ1, FQ2, and FQ3 in Table 10), basic manipulations were clearly considered easier with Tangible. They found it easier to perform explorations using tangibles (FQ4). This also includes Hybrid, since many collection instantiation operations in the MTS task involved the use of pucks.

Tangible interaction was seen to be easier than Touch interaction for moving an item from a collection to a target (FQ6), as required in insertion operations. For deletion operations (FQ7), Touch was given higher preference. They considered that grasping a puck just to perform a deletion interrupted the sequence of operations. However, the overall editing process was considered to be easier in Hybrid, closely followed by Tangible (FQ8). None of the subjects chose Touch, which suggests that dragging items through cluttered conditions is to be avoided when designing a multi-touch tangible user interface. This finding also supports the idea that more complex and general scenarios, with a range of different actions such as exploring, selecting, dragging, etc., are better

Table 10. Final questionnaire summary. Participant counts and the percentage on each question are summarized.

\begin{tabular}{llcccc}
\hline \hline Q & Questions: In general I consider that... & Hybrid & Tangible & Touch & No matter \\
\hline FQ1 & Acquiring a TangiWheel control is easier in... & $8 / 34.8 \%$ & $14 / 60.9 \%$ & $1 / 4.3 \%$ & 0 \\
FQ2 & Moving a TangiWheel control to a target location is easier & $2 / 8.7 \%$ & $16 / 69.6 \%$ & $2 / 8.7 \%$ & $3 / 13 \%$ \\
& in... & & & \\
FQ3 & Rotating a TangiWheel control to a target angle is easier & $1 / 4.3 \%$ & $18 / 78.3 \%$ & $4 / 17.4 \%$ & 0 \\
& in... & & & \\
FQ4 & Exploring items in collections is easier in... & $8 / 34.8 \%$ & $8 / 34.8 \%$ & $5 / 21.7 \%$ & $2 / 8.7 \%$ \\
FQ5 & Selecting items in collections is easier in... & $7 / 30.4 \%$ & $5 / 21.7 \%$ & $11 / 47.8 \%$ & 0 \\
FQ6 & Moving an item from a collection to a target is easier in... & $2 / 8.7 \%$ & $19 / 82.6 \%$ & $2 / 8.7 \%$ & 0 \\
FQ7 & Deletions are easier in... & $1 / 4.3 \%$ & $6 / 26.1 \%$ & $11 / 47.8 \%$ & $5 / 21.7 \%$ \\
FQ8 & Editing a series is easier in... & $11 / 47.8 \%$ & $10 / 43.5 \%$ & 0 & $2 / 8.7 \%$ \\
FQ9 & I explore items more accurately in... & $7 / 30.4 \%$ & $10 / 43.5 \%$ & $5 / 21.7 \%$ & $1 / 4.3 \%$ \\
FQ10 & I carry out the overall selection process quicker ... & $16 / 69.6 \%$ & $4 / 17.4 \%$ & $3 / 13 \%$ & 0 \\
FQ11 & Editing a series is quicker in... & $10 / 43.5 \%$ & $12 / 52.2 \%$ & 0 & $1 / 4.3 \%$ \\
FQ12 & Repetitive manipulations on an item are more effective in... & $1 / 4.3 \%$ & $22 / 95.7 \%$ & 0 & 0
\end{tabular}


suited for hybrid interaction styles.

The participants felt that Hybrid allowed them to perform selections more quickly (FQ10), probably as a consequence of the combination of exploring with pucks and selecting by fingers, as had been shown by the detailed analysis. Hybrid was perceived as effective for editing series (FQ11), although not as fast as Tangible, as could be expected. Finally, the Tangible memory capacity was a positive feature, as repetitive manipulations were clearly considered more effective in the Tangible approach (FQ12).

\section{Conclusions}

Several proposals for handling collections on tabletop interfaces were reported and analyzed. In the course of this analysis, several features were extracted to allow us to describe all the proposals in a similar fashion to facilitate a systematic comparison. The comparison with existing related work reveals that no widget is presently available for tabletop collection management that supports mixed multi-modal input with high levels of resemblance, supporting direct-access and replication of sub-collections in deeply nested structures with spatial compactness, allowing for a large number of collections to be simultaneously manipulated on a shared space by multiple users. From this starting point, this paper presents the specially-designed TangiWheel collection manipulation widget and a description of its implementation on interactive surfaces. This widget supports not only direct finger and tangible interaction by pucks or handlers, but also a hybrid interaction scheme that allows users to combine both input methods as required. Among the different aspects that could be evaluated, the work concentrated on an empirical evaluation to study how the input method (Touch, Tangible or Hybrid) affects performance in the typical interactions involved in manipulating collections using TangiWheel on tabletop displays under different circumstances.

Certain limitations in our experiments and the scope of the results should be pointed out. The experiments were focused on the collections as performed by TangiWheel. This means that the results should be considered in this context, even though the design discussion and results are directly applicable to future development efforts of pie-based widgets to support collection handling with features such as those given in Table 1.

As suggested by Experiment 1, pucks are more effective than dragging and rotational finger-based input when basic acquisition and manipulation actions (such as fine-grained rotations) are performed. However, for exploration actions on collections, gestures based on one-finger rotations are more effective than rotations with pucks. This is mainly due to the lack of interruptions, allowing for a continuous interaction no matter how big the collection. For the same reason it could be expected that a linear touch gesture for exploring large collections would be less effective than our rotational one, but additional experiments would be needed to confirm this hypothesis. However, if explorations are accompanied by highly recurrent selections and frequently moving elements in the 
collections to other areas on the surface, and if the application needs an increasing number of collections to be available on the surface with a non-negligible number of actions for the selection and movement of collections, similar to the conditions in Experiment 3, then the use of pucks for selecting and moving collections and items has advantages over touch-based gestures. No single modality is therefore preferable in all cases and effectiveness varies according to the nature of the application and the actions to be carried out. A mixed input modality, such as that included in TangiWheel, offers advantages over existing tabletop-oriented widgets for collection management in situations in which the best of both methods is required. This is especially the case in applications in which multiple users have to share many large collections and work simultaneously on a shared space.

TangiWheel is now being used in a creative learning environment based on tangible surface interfaces focused on the creation and simulation of virtual ecosystems [23], which require extensive exploring and selection operations. In this context, an empirical exploratory study on teenagers' creativity was reported comparing a digital platform using TangiWheel against a physical tangible-only tabletop. In terms of interaction in a real setting, the digital platform showed higher team co-operation as well as higher complexity levels in the artifacts created. This degree of success would not have been possible with a system other than TangiWheel, especially in the case of teenagers using the digital interface collaboratively, with features such as parallel collection support, view independence, easy hybrid input and direct access to items, etc.

For future work, although it would be interesting to empirically study many of the features that have not been directly faced in the present study, the most important aspect to be explored is that of multi-user support, which would require an experimental design involving four subjects simultaneously manipulating TangiWheel collections. Length, and consequently visual layout, could also be possibly included to complement the present work. Of particular interest would be an experiment specifically designed to explore how well different collection lengths are managed by this proposal and alternatives based on the improvements mentioned in the design discussion, such as showing items near the bridge mark on an extended viewer, providing shortcuts to items in specific positions similar to bookmarks, or even using a linear arrangement approach.

Another line of future research is adding context-awareness to the widget so that its appearance and behavior adapt dynamically to ambient factors, such as other nearby TangiWheel widgets, the available surface space or the most frequently accessed items in the collection.

Acknowledgement Our thanks to the ASIC/Polimedia team for the support in computer hardware. Also thanks to the participants in the study reported here, for their effort and sharing 
their valuable time. Finally, we would also like to acknowledge the useful comments and suggestions made by reviewers throughout the reviewing process, whose dedication has significantly improved this work.

\section{References}

[1] Jordà S. Geiger, G., Alonso, M. Kaltenbrunner, M. The reacTable: exploring the synergy between live music performance and tabletop tangible interfaces. In Proc. TEI’07, Feb. 15-17, 2007, Baton Rouge (LA), USA, ACM Press, pp. 139-146.

[2] Vandoren, P., Van Laerhoven, T., Claesen, L., Taelman, J., Raymaekers, C., Van Reeth, F. IntuPaint: Bridging the gap between physical and digital painting, In Proc. ITS’08, Oct. 1-3, 2008, Amterdam, The Netherlands, ACM Press, pp. 65-72.

[3] Schöning, J., Hecht, B., Raubal, M., Krüger, A., Marsh, M., Rohs, M. Improving interaction with virtual globes through spatial thinking: helping users ask "why?". In Proc. IUI '08, Jan. 13-16, 2008, Canary Islans, Spain. ACM, pp. 129-138.

[4] Fitzmaurice, G.W., Buxton, W. An Empirical Evaluation of Graspable User Interfaces: towards specialized, space-multiplexed input. In Proc. CHI '97, March 22-27, 1997, Atlanta (GA), USA, ACM, pp. 43-50.

[5] Tuddenham, P., Kirk, D., Izadi, S. Graspables -30 - revisited: multi-touch vs. tangible input for tabletop displays in acquisition and manipulation tasks. In Proc. CHI '10, April 10-15, 2010, Atlanta (GA), USA, ACM Press, pp. 2223-2232.

[6] Lucchi, A., Jermann, P., Zufferey G., Dillenbourg, P. An Empirical Evaluation of Touch and Tangible Interfaces for Tabletop Displays. In Proc. TEI 2010, Jan. 25-27, 2010, Boston (MA), USA, ACM. Pp. 177-184.

[7] Fitzmaurice, G.W., Ishii, H., Buxton, W. Bricks: Laying the Foundations for Graspable User Interfaces. In Proc. CHI'95, May. 7-11, 1995, Denver (CO), USA, ACM Press, pp. 442-449,.

[8] Ishii, H., Ullmer, B. Tangible Bits: Towards Seamless Interfaces between People, Bits and Atoms. In Proc. CHI'97, March 22-27, 1997, Atlanta (GA), USA, ACM Press, pp. 234-241.

[9] Ullmer, B., Ishii, H., Glas D. mediaBlocks: physical containers, transports, and controls for online media. In Proc. SIGGRAPH '98, July 19-24, 1998, Orlando (FL), USA, pp. 379-386.

[10]Shen C., Hancock M.S., Forlines C., Vernier F.D., CoR2Ds: Context-Rooted Rotatable Draggables for Tabletop Interaction, In Proc. CHI'05, April 2-7, 2005, Portland (OR), USA, ACM Press, pp. 1781-1784.

[11]Lepinski, G. J., Grossman, T., Fitzmaurice, G. The design and evaluation of multitouch marking 
menus. In Proc. CHI '10, April 10-15, 2010, Atlanta (GA), USA, ACM Press, pp. 2233-2242.

[12]Accot, J. and Zhai, S. Beyond Fitts' law: models for trajectory-based HCI tasks. In Proc.. CHI97, March 22-27, 1997, Atlanta (GA), USA, ACM, pp. 295-302.

[13] Song, H., Kim, B., Lee, B., Seo, J. A comparative evaluation on tree visualization methods for hierarchical structures with large fan-outs. In Proc. CHI '10, April 10-15, 2010, Atlanta (GA), USA, ACM, pp. 223-232.

[14]Bailly, G., Lecolinet, E., Nigay, L. Wave menus: improving the novice mode of hierarchical marking menus. In Proc. INTERACT 2007, Sep. 10-14, 2007, Río de Janeiro, Brazil, Lecture Notes In Computer Science. Springer-Verlag, pp. 475-488.

[15]Zhao, S., Agrawala, M., Hinckley, K. Zone and polygon menus: using relative position to increase the breadth of multi-stroke marking menus.

In Proc. CHI’06, April 24-27, 2006, Montreal, Canada, ACM Press, pp. 1077-1086.

[16]Patten J., Recht B., Ishii H. Interaction techniques for musical performance with tabletop tangible interfaces. In Proc. ACE’06, Jun. 14-16, 2006, Hollywood (CA), USA, article 27.

[17]Weiss M., Jennings R., Khoshabeh R., Borchers J., Wagner J., Jansen Y., Hollan J. D. SLAP widgets: bridging the gap between virtual and physical controls on tabletops. In Proc. CHI '09, April 4-9, 2009, Boston (MA), USA, ACM, pp. 3229-3234.

[18]Hancock, M., Hilliges, O., Collins, C., Baur, D., Carpendale, S. Exploring tangible and direct touch interfaces for manipulating 2D and 3D information on a digital table. In Proc. ITS '09, Nov. 23-25, Banff, Canada, ACM, pp. 77-84.

[19]Hilliges O., Baur D., Butz A. Photohelix: Browsing, Sorting and Sharing Digital Photo Collections. In Proc. Horizontal Interactive $\mathrm{Hu}-$ man-Computer Systems (TABLETOP'07), Oct. 10-12, 2007, Newport, Rhode Island, USA, pp. 87-94.

[20]Hesselmann, T., Flöring, S., Schmidt, M. Stacked Half-Pie menus: navigating nested menus on interactive tabletops. In Proc. ITS'09, Nov. 23-25, 2009, Banff, Canada, ACM Press, pp. $173-180$

[21] Gallardo, D., Jordà, S. Tangible jukebox: back to palpable music. In Proceedings of TEI '10, Jan. 25-27, 2010, Boston (MA), USA, ACM, pp. 199-202.

[22]Fishkin K.. A Taxonomy for Analysis of Tangible Interfaces. In Proc. Personal and Ubiquitous Computing, vol. 8, pp. 347-358, 2004.

[23] Catala, A., Jaen, J., Martinez-Villaronga, A.A., 
Mocholi, J.A., AGORAS: Exploring Creative Learning on Tangible User Interfaces. In Proc. COMPSAC2011, July 18-22, 2011, Munich, Germany, IEEE, pp.326-335.

[24]Catala, A., Garcia-Sanjuan, F., Azorin, J., Jaen, J., Mocholi, J.A., Exploring Direct Communication and Manipulation on Interactive Surfaces to Foster Novelty in a Creative Learning Environment. IJCSRA, 2:1, pp. 15-24, ISSN 2012-9564 (2012).

[25]Catala, A., Jaen, J., van Dijk, B., Jordà, S., Exploring Tabletops as an Effective Tool to Foster Creativity Traits, In Proc. TEI'2012, Feb. 19-22, 2012, Kingston (ON), Canada, to appear.

[26]Hopkins D. Directional Selection is Easy as Pie Menus!, In: The Usenix Association Newsletter, 12, 5(1987).

[27]Microsoft Surface User Experience Guidelines, 26/05/2011, MSDN Library: http://msdn.microsoft.com/en-us/library/ff318692. $\underline{\operatorname{aspx}}$

[28] Maydak, M., Stromer, R., Mackay, H.A., Stoddard, L.T. Stimulus classes in matching to sample and sequence production: The emergence of numeric relations. Research in Developmental Disabilities, 16-3, 179-204, ISSN 0891-4222 (1995).

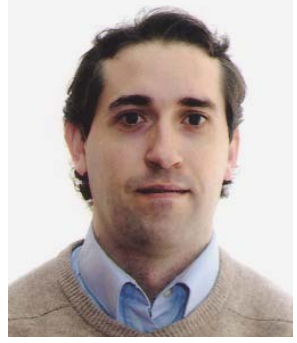

Alejandro Catalá received the degree in Computer Science Engineering from the Universitat Politècnica de València (Spain) in 2006. He received the Best Student Award in Computer Science in 2006 from Social Council at the Universitat Politècnica de València. He also received Master's degree in Software Engineering, Formal Methods, and Information Systems. Now he is a $\mathrm{PhD}$ candidate in the Computer Science programme. His current research interests include interactive and narrative systems for learning and entertainment purposes, surface computing, augmented reality, ubiquitous computing, and evolutionary computation

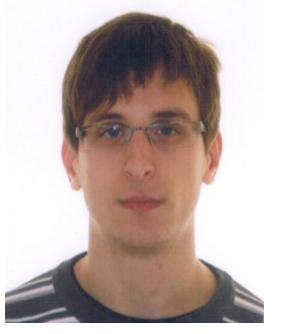

Fernando García-Sanjuan is a student in the last course of Computer Science Engineering programme at the Universitat Politècnica de València. He has been granted with a fellowship from the Ministry of Education to work as a collaborator of the FutureLab team at the ISSI group. Now he is carrying out his final project in the context of tabletop interfaces for learning purposes. 


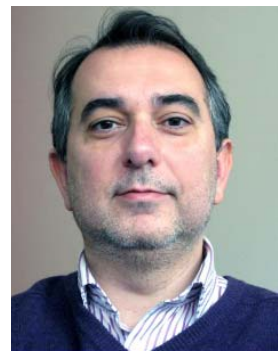

Javier Jaen is head of the FutureLab team, computer science profesor at the Universitat Politècnica de València (Spain), Fulbright scholarship recipient and member of Upsilon Pi Epsilon, International Honour Society for the Computing and Information Disciplines. He has obtained a Master of Science degree from Virginia Tech (USA) and an Advanced Studies Degree from Institut National des Sciences Appliquées de Lyon (France). His research interests include grid computing, ant colony optimization, tangible interfaces and ubiquitous systems. He is the lead researcher of several research projects in collaboration with companies such as Microsoft Research, Telefónica R\&D, Bancaja and America’s Cup Management. He has been awarded the best $\mathrm{PhD}$ dissertation in computer science award at UPV, the award from the Society Commission at UPV 2007 and the eMobility 2003 award.

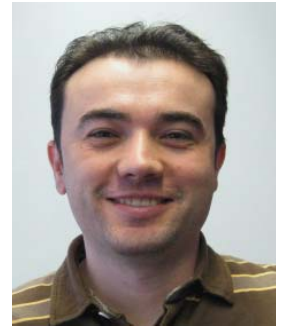

Jose A. Mocholí received his Ph.D. in Computer Science from the Universidad Politécnica de Valencia in 2011. He is currently a researcher in the Department of Information Systems and Computation at the same university. His current research interests include affective computing, evolutionary computation, augmented reality, ubiquitous and distributed computing. 